\title{
Solving quantum master equations with deep quantum neural networks
}

\author{
Zidu Liu $\odot,{ }^{1}$ L.-M. Duan,,${ }^{1, *}$ and Dong-Ling Deng ${ }^{1,2, \dagger}$ \\ ${ }^{1}$ Center for Quantum Information, IIIS, Tsinghua University, Beijing 100084, China \\ ${ }^{2}$ Shanghai Qi Zhi Institute, 41st Floor, AI Tower, 701 Yunjin Road, Xuhui District, Shanghai 200232, China
}

(Received 28 August 2020; accepted 29 January 2022; published 9 February 2022)

\begin{abstract}
Deep quantum neural networks may provide a promising way to achieve a quantum learning advantage with noisy intermediate-scale quantum devices. Here, we use deep quantum feed-forward neural networks capable of universal quantum computation to represent the mixed states for open quantum many-body systems and introduce a variational method with quantum derivatives to solve the master equation for dynamics and stationary states. Owning to the special structure of the quantum networks, this approach enjoys a number of notable features, including an efficient quantum analog of the back-propagation algorithm, resource-saving reuse of hidden qubits, general applicability independent of dimensionality and entanglement properties, as well as the convenient implementation of symmetries. As proof-of-principle demonstrations, we apply this approach to both one-dimensional transverse field Ising and two-dimensional $J_{1}-J_{2}$ models with dissipation, and show that it can efficiently capture their dynamics and stationary states with a desired accuracy.
\end{abstract}

DOI: 10.1103/PhysRevResearch.4.013097

\section{INTRODUCTION}

Recent developments in quantum hardware have reached a stage where quantum devices with tens to hundreds of controllable qubits will soon become available [1-6]. An important milestone for these so-called noisy intermediate-scale quantum (NISQ) devices [7] is Google's demonstration of quantum supremacy with 53 programmable superconducting qubits [1]. This opens up exciting possibilities of utilizing NISQ devices to tackle challenging real-world problems that are beyond the capacity of any classical computers [8]. Along this line, a variety of hybrid quantum-classical approaches have been pushed forward, including the variational quantum eigensolver (VQE) [9-12], the quantum approximate optimization algorithm (QAOA) [13-15], quantum classifiers [16-18], quantum generative adversarial networks [19-21], etc. In this paper, based on deep quantum feed-forward neural networks [22] (DQFNNs) we introduce a variational method with quantum derivatives to obtain the dynamics and stationary states for open quantum many-body systems by solving the Lindblad master equations (see Fig. 1 for a pictorial illustration).

Every quantum system is inevitably coupled to its surrounding environment. In most cases, the coupling is of Markovian type and the dynamics of open quantum systems are governed by the Lindblad quantum master equation [23].

\footnotetext{
*1mduan@tsinghua.edu.cn

†dldeng@tsinghua.edu.cn
}

Published by the American Physical Society under the terms of the Creative Commons Attribution 4.0 International license. Further distribution of this work must maintain attribution to the author(s) and the published article's title, journal citation, and DOI.
Thus, solving this master equation plays a key role in studying open quantum systems. However, for quantum many-body systems this turns out to be a formidable challenge due to the exponential growing of the Hilbert space dimension with the size of the system. To combat this challenge, a number of prominent numerical approaches based on classical computers have been developed, such as those using tensor network representations [24-31] or quantum Monte Carlo methods [32,33]. More recently, machine-learning inspired approaches based on artificial neural networks [34-39], especially the restricted Boltzmann machine (RBM), have also been introduced to solve the quantum master equation [36-39]. Each of these approaches bears its own advantages and disadvantages, and the choice of which one to use is problem specific. For instance, tensor-network approaches are typically very effective for one-dimensional (1D) systems involving small entanglement, but may face pronounced difficulties in higher dimensions or in situations where massive entanglement comes into play [40,41]. Quantum Monte Carlo methods rely on efficient sampling of physical configurations [42] and are effective for certain open quantum systems [32,33], yet could suffer from a severe sign problem that is common in simulating dissipative dynamics [43]. The neural-network approaches [36-39] are generally applicable to high-dimensional systems and entanglement is not a limiting factor [44]. However, as in most machine-learning tasks [45], their performance depends crucially on the suitable tuning of hyperparameters. In fact, the capability and limitation of neural-network methods in solving quantum many-body problems still remain largely unclear, and related studies are at the research forefront.

Here, inspired by the classical neural-network approaches and the exciting experimental progress in developing NISQ devices, we propose a variational DQFNN method to solve the quantum master equations. We note that variational algorithms running on NISQ devices for open quantum systems 


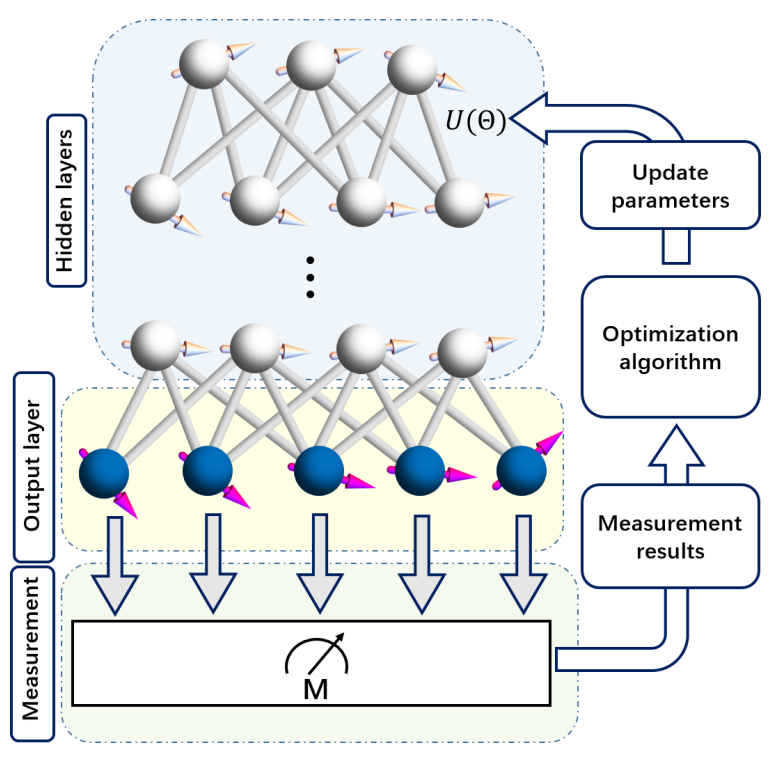

FIG. 1. Schematic illustration of the DQFNN (deep quantum feed-forward neural network) method in solving quantum master equations. This DQFNN has $d$ layers in total, with an output (bottom) layer that indicates the target quantum system, and $d-1$ hidden layers that simulate the environment and conduct interaction in the target quantum system. A quantum perceptron is defined as an arbitrary unitary operator acting on qubits from neighboring layers [22], and the DQFNN is parametrized by the product of these unitary operators. We use the output state as the variational ansatz state of a quantum open system. The qubits in the output layer are measured and the measurement results are used by a quantum optimization algorithm to update the network parameters.

have been discussed in the literature [46-49], but to the best of our knowledge most existing works focus on straightforward variational quantum circuits that may suffer from the notorious barren plateau (i.e., vanishing gradient) problem [50-58]. We utilize a deep quantum neural network, which is arranged in layers and convenient for the quantum analog of the backpropagation algorithm, to serve as the ansatz density state of the open quantum system. We adopt a hybrid quantumclassical stochastic reconfiguration (SR) algorithm [59] to variationally solve the quantum master equation in the Lindblad form to obtain the dynamics and steady state. As a result of the special structure of the quantum networks, our approach allows for convenient implementation of translational symmetry and resource-saving reuse of hidden qubits. Besides, our optimization algorithm is different with the straightforward gradient descent based method in [52], where the existence of barren plateaus has been proved for global loss functions with the DQFNN structure. In our optimization algorithm, owing to the real-time evolution based quantum SR algorithm, which simulates the real and physical quantum dynamics, the trainability of our model depends crucially on the physical properties of the problem (e.g., the Liouvillian gap). Thus, our approach could circumvent the barren plateau problem. In addition, it works for generic open quantum many-body systems, independent of dimensionality, the amount of entanglement involved, and the specific forms of interaction and dissipation. We benchmark our approach with both dissipative $1 \mathrm{D}$ transverse field Ising and $2 \mathrm{D} J_{1}-J_{2}$ models.

\section{THE GENERAL PROBLEM AND DQFNN STRUCTURE}

The dynamics of the density matrix of an open system is controlled by the quantum master equation, which takes the form

$$
\frac{d \rho}{d t}=\mathcal{L} \rho=-i[H, \rho]-\sum_{i} \frac{\gamma_{i}}{2}\left(\left\{F_{i}^{\dagger} F_{i}, \rho\right\}-2 F_{i} \rho F_{i}^{\dagger}\right),
$$

where $\rho$ is the density matrix of the system, $H$ is the Hamiltonian governing the unitary part of the dynamics, $F_{i}$ are the jump operators describing the dissipative processes induced by the environment with dissipative rate denoted by $\gamma_{i}$, the curly bracket represents the anticommutator, and $\mathcal{L}$ is the Liouvillian superoperator [23].

We use the final output state of a quantum neural network, the recently proposed DQFNN in particular [22], to serve as the variational ansatz for the density state of the open quantum system. The network structure is illustrated in Fig. 1. Each node represents a qubit, and a quantum perceptron is an arbitrary unitary operator acting on several qubits from neighboring layers. Information propagates from the first hidden (top) layer to the output (bottom) layer. At the $i$ th layer, the output state $\rho_{\mathrm{o}}^{(i)}$ is determined by

$$
\rho_{\mathrm{o}}^{(i)}=\operatorname{Tr}_{i-1}\left[\mathbf{U}\left(\rho_{\mathrm{o}}^{(i-1)} \otimes\left|\psi^{(i)}\right\rangle\left\langle\psi^{(i)}\right|\right) \mathbf{U}^{\dagger}\right],
$$

where $\rho_{\mathrm{o}}^{(i-1)}$ is the output state of the $(i-1)$ th layer (which serves as the input state for the $i$ th layer), $\left|\psi^{(i)}\right\rangle\left\langle\psi^{(i)}\right|$ denotes the initial state of the $i$ th layer, $\mathbf{U}=\otimes_{j=0}^{M} U_{i, j}$ represents the total unitary operation between the two layers with $U_{i, j}$ denoting the $j$ th perceptron at the $i$ th layer, and $\operatorname{Tr}_{i-1}$ denotes a partial trace of the $(i-1)$ th layer. Equation (2) may also be regarded as a mapping $\mathcal{M}$ from $\rho_{\mathrm{o}}^{(i-1)}$ to $\rho_{\mathrm{o}}^{(i)}$, and the whole network features a mapping-composition structure, which is essential for the back-propagation algorithm [45]:

$$
\rho_{\mathrm{o}}^{(d)}=\mathcal{M}^{d}\left(\cdots \mathcal{M}^{1}\left(\left|\psi^{(1)}\right\rangle\left\langle\psi^{(1)}\right|\right) \cdots\right) .
$$

Here, $\left|\psi^{(1)}\right\rangle\left\langle\psi^{(1)}\right|$ denotes the initial state of the first hidden layer, and we suppose that the network has $d$ layers in total. We mention that this quantum network is universal [22] in the sense that it can map any input density state to an arbitrary output state due to its vast flexibility in designing the qubit perceptrons. Here, we focus on two-input one-output qubit perceptrons for simplicity and experimental feasibility.

\section{THE GENERAL RECIPE}

We solve the following optimization problem variationally:

$$
\min _{\Theta}\left\|\partial_{t} \rho_{\mathrm{o}}^{(d)}(\Theta)-\mathcal{L} \rho_{\mathrm{o}}^{(d)}(\Theta)\right\|_{\mathrm{FS}},
$$

where $\Theta$ collectively denotes all the parameters used to describe the qubit perceptrons (unitary gates) and the FubiniStudy (FS) norm is used to measure the distance. For convenience, we omit the labels and rewrite the density matrix $\rho_{\mathrm{o}}^{(d)}$ as a vector $\vec{\rho}$. Similarly to the SR algorithm in solving the quantum master equation based on the variational RBM representation [36,38], the above minimization problem results 
in the following system of equations:

$$
\sum_{\nu} S_{\mu, \nu} \dot{\Theta}_{\nu}=f_{\mu}
$$

where $\Theta_{\nu}$ denotes the $\nu$ th parameter, and

$$
\begin{aligned}
S_{\mu, \nu} & =\operatorname{Re}\left(\frac{\partial \vec{\rho}^{\dagger}}{\partial \Theta_{\mu}} \frac{\partial \vec{\rho}}{\partial \Theta_{\nu}}\right)-\operatorname{Re}\left[\left(\frac{\partial \vec{\rho}^{\dagger}}{\partial \Theta_{\mu}} \vec{\rho}\right)\left(\vec{\rho}^{\dagger} \frac{\partial \vec{\rho}}{\partial \Theta_{\nu}}\right)\right], \\
f_{\mu} & =\operatorname{Re}\left(\frac{\partial \vec{\rho}^{\dagger}}{\partial \Theta_{\mu}} \mathcal{L} \vec{\rho}\right)-\operatorname{Re}\left[\left(\frac{\partial \vec{\rho}^{\dagger}}{\partial \Theta_{\mu}} \vec{\rho}\right)\left(\vec{\rho}^{\dagger} \mathcal{L} \vec{\rho}\right)\right] .
\end{aligned}
$$

We initialize the parameters to be small random values and update them iteratively according to the the following rule:

$$
\Theta^{\text {new }}=\Theta^{\text {old }}+\lambda S^{-1} \vec{f},
$$

where $\lambda$ is a hyperparameter (called the learning rate in the machine learning literature) introduced to modulate the convergence of the iteration process.

In real experiments, both $S$ and $\vec{f}$ might be obtained from measurements of observables or their linear combinations (see Appendix C). Then, by using Eq. (8) we update the parameters of the quantum neural network iteratively and obtain the dynamics of the open system consequently. Classical simulation of this experimental setup is costly. Here we propose a classical-quantum hybrid algorithm, where the measurement is based on the Monte Carlo sampling on the elements of the output density matrix, which requires fewer number of qubits (details in Appendix B). At the long-time limit, the neural network state converges to the steady state. In our method, inside each perceptron the three qubits parameterized operator $U$ can be parametrized with respect to the Hermitian generator:

$$
U=e^{-i \sum_{\alpha_{m}, \alpha_{n}, \beta_{j}} \Theta_{\alpha_{m}, \alpha_{n}, \beta_{j}} \sigma^{\alpha_{m}} \otimes \sigma^{\alpha_{n}} \otimes \sigma^{\beta_{j}}},
$$

where the parameter $\Theta_{\alpha_{m}, \alpha_{n}, \beta_{j}}$ denotes the unitary coupling operator among $m$ th and $n$th qubits in the $i$ th layer, and $j$ th qubits in the $(i+1)$ th layer. We expand our unitary operator in the pauli basis $\sigma^{\alpha_{m}} \otimes \sigma^{\alpha_{n}} \otimes \sigma^{\beta_{j}}$, where the indexes $\alpha_{m}, \alpha_{n}, \beta_{j}=0, x, y, z$ indicate the identity and Pauli matrices $I, \sigma^{x}, \sigma^{y}, \sigma^{z}$. As discussed in [22], a striking feature of this DQFNN approach is that the variational parameters can also be calculated layer by layer, and at any given time we need only access to two layers. This will greatly reduce the memory requirements of the algorithm and enables a resource-saving reuse of the qubits.

\section{NUMERICAL SIMULATIONS}

We apply the introduced DQFNN approach to two concrete models to benchmark how it works. The first example involves the dissipative transverse field Ising model in one dimension:

$$
\frac{d \rho}{d t}=-i[H, \rho]-\frac{\gamma}{2} \sum_{j}\left[\left\{\sigma_{j}^{+} \sigma_{j}^{-}, \rho\right\}-2 \sigma_{j}^{-} \rho \sigma_{j}^{+}\right],
$$

where $H=J \sum_{j} \sigma_{j}^{z} \sigma_{j+1}^{z}+h \sum_{j} \sigma_{j}^{x}, \gamma$ denotes the dissipation rate, and $\sigma_{j}^{ \pm}=\frac{1}{2}\left(\sigma_{j}^{x} \pm i \sigma_{j}^{y}\right)$, with $\sigma_{j}^{x, y, z}$ being the Pauli matrices for the site $j$. The first term in the Hamiltonian represents the $z$-component spin-spin interaction in the longitudinal direction with strength $J$ and the second term denotes a local (a)

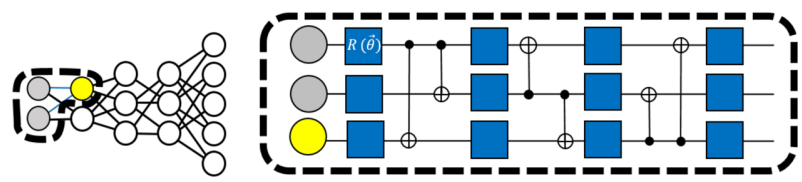

(b)

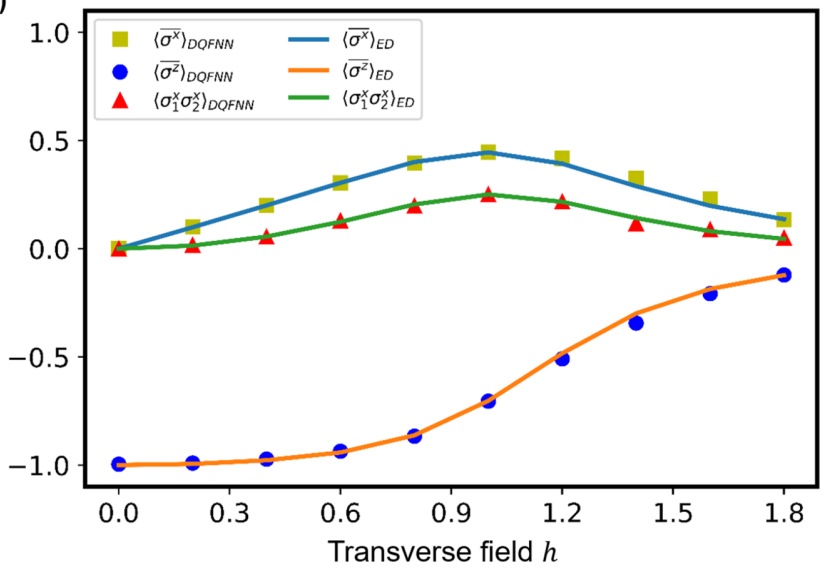

FIG. 2. (a) A DQFNN with layer structure $(2,2,3,3,5)$ is used to solve the dissipative transverse field Ising model [see Eq. (10)]. Here, each quantum perceptron consists of a unitary acting on two-input and one-output qubits and this unitary is parametrized by a simple quantum circuit (black-dotted box), which contains twelve Euler rotations and six controlled-not gates. (b) Numerical results for the steady state of the dissipative Ising model with five spins. The expectation values of three different observables $\overline{\sigma^{x}}=\frac{1}{5}\left(\sum_{k} \sigma_{k}^{x}\right), \overline{\sigma^{z}}=$ $\frac{1}{5}\left(\sum_{k} \sigma_{k}^{z}\right)$, and $\sigma_{1}^{x} \sigma_{2}^{x}$ are calculated by both the DQFNN and exact diagonalization (ED) methods. Here, we set $J=1$ as the energy unit and the dissipation ratio is $\gamma=1$. For the DQFNN approach, the learning rate $\lambda$ is chosen to be $\lambda=0.01 \times 0.999^{N_{s}}$, where $N_{s}$ is the number of steps.

uniform magnetic field with strength $h$ along the transverse direction. We mention that the steady state of this model has been studied in Ref. [37] with a classical neural-network (i.e., RBM) approach. Here, instead we apply the DQFNN method to obtain both the dynamics and steady state. The quantum neural-network structure is shown in Fig. 2(a). The system size is $N=5$ and the quantum network has five layers and contains 15 qubits in total. Each perceptron consists of three qubits coupled by a series of unitary gates as shown by the quantum circuit in the dotted box in Fig. 2(a). For this structure, considering an $L$-layer DQFNN with $N_{p}$-perceptrons, the total number of entangling two-qubit gates scales as $6 \times N_{p}=$ $6 \sum_{i=2}^{L} N_{i}$, where $N_{i}$ is the number of qubits in the $i$ th layer. Hence, the entangling gates grow linearly with respect to the system size. We assume a periodic boundary condition and implement translational symmetry to reduce the number of parameters. In addition, owing to the special structures of the quantum perceptrons, the derivatives of the density states with respect to the parameter $\Theta_{v}$ can be obtained by [60]

$$
\frac{\partial \rho\left(\Theta_{v}\right)}{\partial \Theta_{\nu}}=\frac{1}{2}\left[\rho\left(\Theta_{v}+\frac{\pi}{2}\right)-\rho\left(\Theta_{v}-\frac{\pi}{2}\right)\right] .
$$

We mention that the derivatives in Eq. (11) is exact, in sharp contrast to the finite-difference method $\frac{\partial \rho\left(\Theta_{v}\right)}{\partial \Theta_{v}} \approx \frac{1}{2 \delta_{f}}\left[\rho\left(\Theta_{v}+\right.\right.$ $\left.\left.\delta_{f}\right)-\rho\left(\Theta_{v}-\delta_{f}\right)\right]$, that may inevitably induce a small 

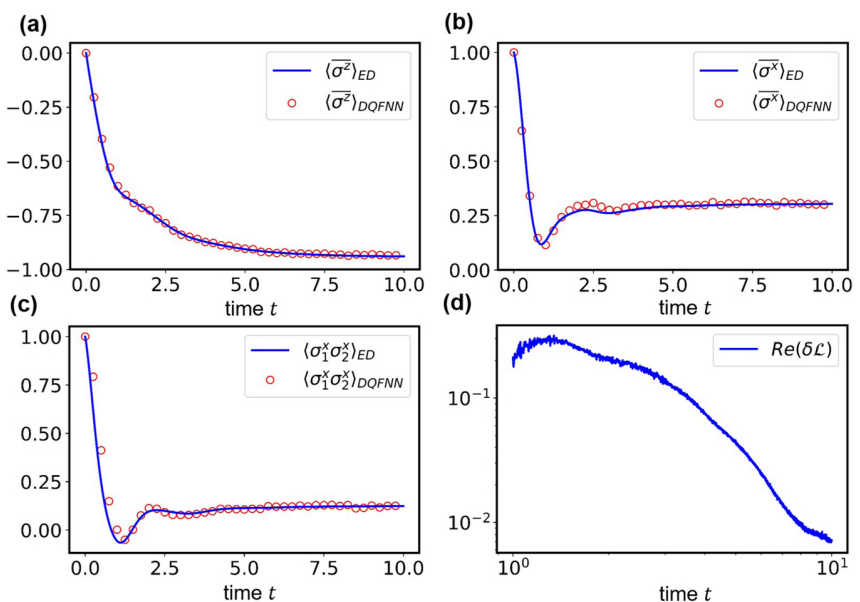

FIG. 3. Numerical results for the time dynamics of the dissipative Ising model with five spins under the periodic boundary condition. The time step is chosen to be $5 \times 10^{-3}$ and the sampling size for every step is $5 \times 10^{4}$. Here we plot each point for every 50 steps. (a), (b), and (c) plot the time dependences of $\overline{\left\langle\sigma^{z}\right\rangle}, \overline{\left\langle\sigma^{x}\right\rangle}$, and $\left\langle\sigma_{1}^{x} \sigma_{2}^{x}\right\rangle$, respectively. (d) plots the real and imaginary parts of $\delta \mathcal{L}$, which measures the convergence to the steady state.

differential error $\left[\sim O\left(\delta_{f}^{2}\right)\right]$. This might be important for the convergence of the optimization procedure [61].

With classical computers, we numerically simulate the quantum neural networks and the whole process of using the DQFNN approach to obtain the dynamics and steady state for the dissipative Ising model defined in Eq. (10). We start with a DQFNN state where all the constituted qubits are initially set to be $|+\rangle=(|0\rangle+|1\rangle) / \sqrt{2}$ and all the involved parameters are initially set to be a small value near zero. Then the parameters are updated according to the optimization procedures shown in Eq. (8). Partial results are shown in Figs. 2(b) and 3. In Fig. 2(b), we compute the steady state with varying strength of the transverse field $h$ through the DQFNN approach. We plot the magnetization values $\overline{\left\langle\sigma^{x, z}\right\rangle}$ and correlations $\left\langle\sigma_{1}^{x} \sigma_{2}^{x}\right\rangle$ for the stationary state, and compare them with the ED results. It is clear that the DQFNN results match the ED results excellently. In Fig. 3, we consider the dynamics of our DQFNN during the optimization process with fixed $h=0.6$. In Figs. 3(a)-3(c), we plot the dynamic evolutions of magnetization values $\overline{\left\langle\sigma^{x, z}\right\rangle}$ and correlations $\left\langle\sigma_{1}^{x} \sigma_{2}^{x}\right\rangle$, and compare their corresponding results obtained by ED. From these figures, it is evident that the DQFNN results match the ED results (with relative error smaller than $10^{-2}$ ) and converge to the corresponding values for the stationary state at long time, where $|\delta \mathcal{L}|$ approaches zero as shown in Fig. 3(d).

Our second example concerns the dissipative $J_{1}-J_{2}$ model defined on a square-lattice in 2D with Hamiltonian

$$
H=J_{1} \sum_{\langle i, j\rangle} \sigma_{i}^{z} \sigma_{j}^{z}+J_{2} \sum_{\langle\langle i, j\rangle\rangle} \sigma_{i}^{z} \sigma_{j}^{z}+h \sum_{i} \sigma_{i}^{x},
$$

where the first (second) term represents the nearest-neighbor (next-nearest-neighbor) $z$-component spin-spin interaction with strength $J_{1}\left(J_{2}\right)$. The dissipation considered here is the same as that for the 1D Ising case [see Eq. (10)]. For this
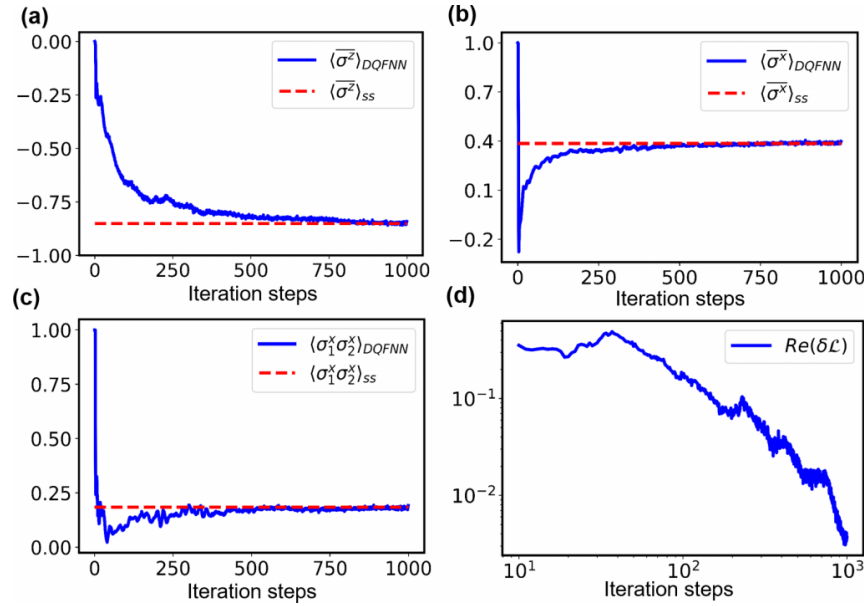

FIG. 4. Numerical results for the dissipative 2D $J_{1}-J_{2}$ model. Here, the simplest two-by-two square lattice is considered and we use a DQFNN with layer structure $(4,4,4)$ to obtain the results. The parametrization of the DQFNN is similar to the case for the 1D dissipative Ising model. (a), (b), and (c) show respectively $\overline{\left\langle\sigma^{z}\right\rangle}, \overline{\left\langle\sigma^{x}\right\rangle}$, and $\left\langle\sigma_{0}^{x} \sigma_{1}^{x}\right\rangle$ as a function of iteration steps with the dissipation rate chosen to be $\gamma=1.0$. The red-dotted lines represent their corresponding values for the steady state (SS) obtained from exact diagonalization. (d) The real part of $\delta \mathcal{L}$, which converges to zero after around 1000 iteration steps $\left[\operatorname{Re}(\delta \mathcal{L})<10^{-2}\right]$, indicating that the output state of the DQFNN indeed converges to the steady state.

Hamiltonian, it is easy to observe that there is a geometric frustration in the antiferromagnetic state due to the competition between the first and second terms. For simplicity, we set $J_{1}=1$ as the energy unit, $J_{2}=\frac{1}{2} J_{1}, h=J_{1}$, and the dissipation rate $\gamma=J_{1}$. Our numerically simulated results from the DQFNN approach are shown in Fig. 4. In Figs. 4(a)-4(c), we plot the magnetization values $\overline{\left\langle\sigma^{x, z}\right\rangle}$ and the correlation function $\left\langle\sigma_{1}^{x} \sigma_{2}^{x}\right\rangle$ as a function of iteration steps. As shown, it is clear that all these quantities converges nicely to their corresponding exact values for the steady state (after 1000 iterations, their relative errors are all smaller than $10^{-2}$ ). This indicates that after around 1000 iterations the output state $\rho_{\mathrm{o}}^{(d)}$ of the DQFNN indeed converges to the steady state, which is also clearly manifested in Fig. 4 as $\delta \mathcal{L}$ converges to zero [after 1000 iterations, $\operatorname{Re}(\delta \mathcal{L})$ becomes less than $10^{-2}$ ]. In addition, we mention that our DQFNN approach is robust to experimental noises (see Appendix E).

We remark that the accuracy of our DQFNN results can be improved in a number of ways, including increasing the number of layers of the quantum neural network or the sampling size at each iteration step, tuning hyperparameters, and designing more appropriate quantum perceptrons, etc. Due to limited classical computation resources, in this paper we only carry out simulations for small systems. However, this will not be a problem if quantum devices are used in practice. As discussed in Appendix F, the time complexity of the DQFNN approach is about $O\left(N^{3}\right)$, which indicates its scalability to larger systems. We also stress two possible intriguing advantages of the DQFNN approach compared with the RBM method [36-39]. First, DQFNNs have notably stronger representation power. For certain distributions, the RBM representation may require an exponential number of parameters, 
whereas for the DQFNN approach it only needs a polynomial number of parameters, as proved in Appendix A from the computational complexity theory perspective. Second, the RBM method is entirely classical and the gradients that are crucial in updating parameters rely on efficient Monte Carlo sampling. In contrast, for the DQFNN method the gradients can be obtained directly from measurements of observables in experiment (see Appendix C). This might lead to an advantage in computational cost for certain problems where no efficient Monte Carlo sampling scheme is available, such as strongly frustrated systems with a severe sign problem.

\section{CONCLUSION}

We have introduced a deep quantum neural-network method to solve Lindblad master equations for open quantum many-body systems. Through concrete examples in both 1D and $2 \mathrm{D}$, our results demonstrated that both the dynamics and stationary states of such systems can be efficiently obtained via this method with a desirable accuracy. Due to the special structures of DQFNNs, our approach is generally applicable to high-dimensional systems and is independent of the amount of entanglement involved. In addition, it is robust to experimental imperfections, and allows a convenient implementation of translational symmetry and a resource-saving reuse of qubits. We implement a time evolution based algorithm, which may circumvent the barren plateaus problem. This classical-quantum hybrid approach is particularly suitable for running on NISQ devices in the near future.

The source code for this work will be available on Github.

\section{ACKNOWLEDGMENTS}

We thank Yuanhang Zhang, Sirui Lu, and Xun Gao for valuable discussions, and Marco Cerezo, Leonardo Banchi, and Suguru Endo for helpful communications. This work was supported by the National Natural Science Foundation of China (Grant No. 12075128), Tsinghua University, and the Ministry of Education of China. D.-L.D. also would like to acknowledge additional support from the Shanghai Qi Zhi Institute.

\section{APPENDIX A: REPRESENTATION POWER OF THE DQFNN}

In our DQFNN, the perceptron with two inputs and one output contains three qubits and a series of unitary gates, which belong to the $S U\left(2^{3}\right)$ group with a universal form:

$$
U_{i, j}=\exp \left[-i \sum_{\alpha_{1}, \alpha_{2}, \alpha_{3}} \frac{\Theta_{i, j, \vec{\alpha}}}{2} \sigma^{\alpha_{1}} \otimes \sigma^{\alpha_{2}} \otimes \sigma^{\alpha_{3}}\right],
$$

where $\Theta_{i, j, \vec{\alpha}}$ denote the parameters for the $j$ th perceptron in the $i$ th layer. The index vector $\alpha_{1,2,3}$ takes $0, x, y, z$ to indicate the identity and Pauli matrix $I, \sigma^{x}, \sigma^{y}, \sigma^{z}$ respectively. Hence, to fully describe a quantum perceptron in this case needs 64 parameters at most, which is a constant number when the system size scales up.

To show the representation power of our DQFNN, let us firstly prove that DQFNN is universal. As shown in Fig. 5(a), an arbitrary single-qubit gate can be applied on the blue and

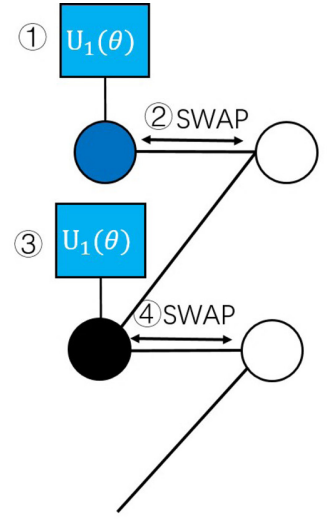

(a)

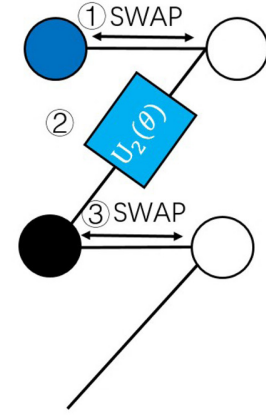

(b)
FIG. 5. Illustration that the DQFNN can represent any singlequbit gate and the nearest two-qubit operation. (a) shows that any single-qubit gate can be generated. The blue and the black circuits represent the two target qubits and the blue boxes $U_{1}(\theta)$ represent the arbitrary single-qubit gate, which can be applied respectively in their perceptrons. The swap gate is applied in their perceptron with the white circuit qubit in the state $\frac{1}{\sqrt{2}}|0\rangle+|1\rangle$. After tracing out the previous layer, we obtain the output density matrix with a single-qubit gate applied. (b) shows that any nearest two-qubit gate can be achieved in our DQFNN: in the first perceptron swap the state of the blue qubit to the next layer and then apply the two-qubit gate $U_{2}(\theta)$, and in the second perceptron swap the black qubit to the next layer. We can obtain the output density matrix with the two-qubit gate applied.

black qubits. We can apply the swap gate in their corresponding perceptron with the white qubit, which transfers the single-qubit operation to the next layer. In Fig. 5(b), we can show that an arbitrary nearest two-qubit gate can be applied between the white qubits in the next layer. As in the first perceptron, we first apply the swap gate between the blue qubit and the first white qubit in the next layer. Then, an arbitrary two-qubit gate is applied between the first white qubit and the black qubit. In the second perceptron, we apply the second swap operation, and realize the arbitrary nearest twoqubit operation.

Then let us prove that any two-qubit gate and single-qubit gate can be calculated by a polynomial number of perceptrons. Consider a DQFNN with $L-1$ layers. We add an additional layer with the same number of qubits as the $(L-1)$ th layer at the end of our networks. For a given output density matrix $\rho^{L-1}$ at the $(L-1)$ th layer, arbitrary two-qubit gate $U_{i, j}$ can be applied in the following ways. As shown in Fig. 6, without the loss of generality, we assume the two-qubit gate is applied on the first and $k$ th qubits, which we denote as black and blue qubits respectively. We apply a series of swap operations in Figs. 6(a) and 6(b) to transfer the black qubit in the $(L-1)$ th layer to the perceptron that contain the blue qubit. In Figs. 6(c) and 6(d) we apply the two-qubit unitary gate operation and swap the blue and black qubits in the perceptron. Then, similarly to our previous operations, in Fig. 6(e) we transfer the state of the black qubit to the first qubit in the $L$ th layer. It can be seen that these operations can also transfer the rest of the qubits in the $(L-1)$ th to their corresponding positions in 


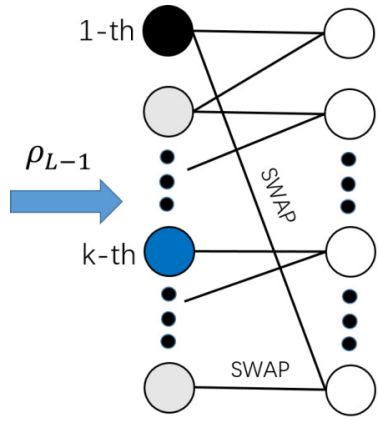

(a)

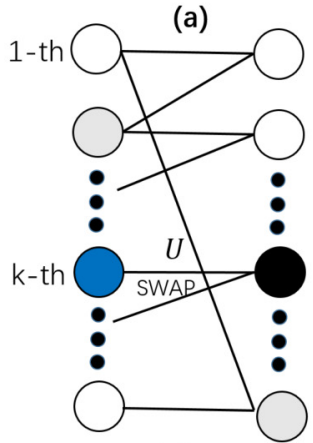

(c)

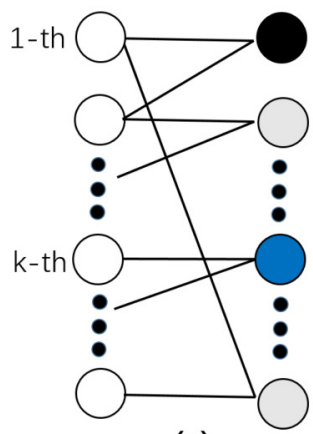

(e)

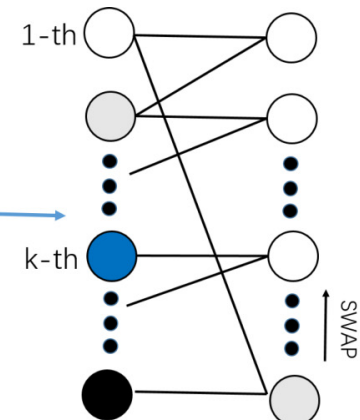

(b)

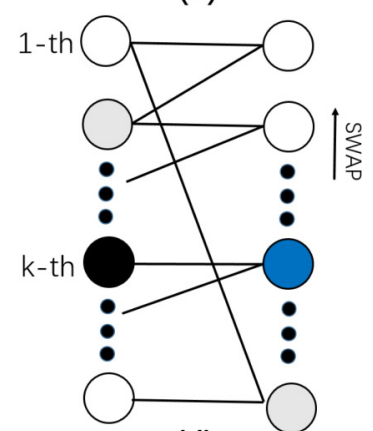

(d)

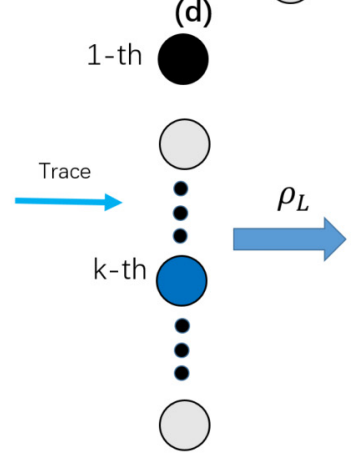

(f)
FIG. 6. Demonstration of implementing an arbitrary unitary operation between two arbitrary qubits in the same layer by applying a series of the unitary gate from (a) to (e). Considering an input density matrix $\rho^{L-1}$ in the $(L-1)$ th layer, we denote the two target qubits by black and blue discs, respectively. Without loss of the generality, we take the first and the $k$ th qubit as the target qubits. The rest of the qubits in the $(L-1)$ th layer are drawn in grey, and the qubits in the $L$ th layer are drawn in white and initialized to $\frac{1}{\sqrt{2}}(|0\rangle+|1\rangle)$. (a) Using two swap gates to transfer the black qubit to the last qubit in the $(L-1)$ th layer. Consequently, the last qubit in the $(L-1)$ th layer is swapped to the corresponding position in $L$ th layer as shown in (b). Repeat this operation until the black qubit and the blue qubit are in the same perceptron, as shown in (c), and we apply the two-qubit unitary gate. After that, in (d), we apply a swap gate between the states of the black qubit and the blue qubit. Then we repeat the swap operations similarly as in (b) until we transfer the black qubit to the $L$ th layer at its corresponding position. It is easy to find that, after these operations, the rest of the qubits in the $(L-1)$ th layer have been swapped to the corresponding position in the $L$ th layer, and the states of qubits in the $(L-1)$ th layer are all taken as $\frac{1}{\sqrt{2}}(|0\rangle+|1\rangle)$ in (e). After tracing out the $(L-1)$ th layer, we obtain the output density matrix in the $L$ th layer with an arbitrary two-qubit unitary gate acting on the two target qubits (f). the $L$ th layer [namely, the $i$ th qubit in the $(L-1)$ th layer is transferred to $i$ th qubit in the $L$ th layer]. As $(L-1)$ th layer and the $L$ th layer now are decoupled from each other, tracing out the qubits in the $(L-1)$ th layer will obtain the result of applying the arbitrary two-qubit gate to the output density matrix $U_{i, j} \rho^{L} U_{i, j}^{\dagger}$. Thus, arbitrary operations can be applied by our DQFNN as long as we add enough layers, where the representation of the output density matrix of the DQFNN is universal. We mention that although our network can be really deep, the depth of the circuit that is applied to each qubit is still a constant number because of our special structure, so that the growth of the DQFNN does not need to consider the effect of qubit decoherence time.

The above proof shows that our DQFNN is universal, and arbitrary two-qubit and one-qubit gates can be applied by a polynomial number of perceptrons. We can deduce that, in computational complexity language, the complexity of sampling the output of our DQFNN is BQP [62]. Like the theorem in [63], the complexity of post-BQP = PP, while for classical computing the $\mathrm{BPP}$ is contained in the $\mathrm{NP}^{\mathrm{NP}}$ and the post-BPP is contained in the $\mathrm{p}^{\mathrm{NP}}{ }^{\mathrm{NP}}[64]$. The Toda theorem shows that $\mathrm{PH} \subseteq \mathrm{p}^{\mathrm{PP}}[65]$. These theorems can lead to a series of containment relationship, namely, $\mathrm{P}^{\text {post-BPP }} \subseteq \mathrm{PH} \subseteq \mathrm{P}^{\mathrm{PP}}=\mathrm{P}^{\text {post-BQP }}$.

Then we will prove that if the output distribution that is generated by our DQFNN can be classically simulated with a multiplicative error, the computational complexity polynomial hierarchy will collapse to the third level. Here we adopt the idea of the proof in $[66,67]$. The multiplicative error between the output probability distribution of the DQFNN and a classical simulator is defined as

$$
\frac{1}{\delta} q\left(x_{l}, x_{r}, \vec{s}_{l}, \vec{s}_{r}\right) \leqslant P\left(x_{l}, x_{r}, \vec{s}_{l}, \vec{s}_{r}\right) \leqslant \delta q\left(x_{l}, x_{r}, \vec{s}_{l}, \vec{s}_{r}\right) .
$$

Here, we define the probability distribution of a classical simulator as $P\left(x_{l}, x_{r}, \vec{s}_{l}, \vec{s}_{r}\right)$, and the output distribution of the DQFNN as

$$
\begin{aligned}
q\left(x_{l}, x_{r}, \vec{s}_{l}, \vec{s}_{r}\right) & =\frac{1}{c}\left|\left\langle x_{l}, x_{r}, \vec{s}_{l}, \vec{s}_{r} \mid \vec{\rho}^{o}\right\rangle\right|^{2} \\
& =\frac{1}{c}\left|\left\langle x_{l}, \vec{s}_{l}\left|\rho^{o}\right| x_{r}, \vec{s}_{r}\right\rangle\right|^{2},
\end{aligned}
$$

where $c$ is a normalization constant, $\rho^{o}$ is the output density matrix of our DQFNN, and $x_{l} / x_{r}\left(\vec{s}_{l} / \vec{s}_{r}\right)$ is the left/right vector configuration of the first (remaining) qubit(s). The first equation uses the Choi-Jamiolkowski isomorphism [68] to map the density matrix to a vector form. We design the DQFNN network with post-selection (post-DQFNN) as taking the first qubit in the output layer as the measurement qubit and taking the rest of the qubits as the post-selection qubits. The probability of the post-DQFNN can be written as

$$
q\left(x_{l}, x_{r}\right)=\frac{q\left(x_{l}, x_{r}, \overrightarrow{s_{l}^{\prime}}, \overrightarrow{s_{r}^{\prime}}\right)}{q\left(\overrightarrow{s_{l}^{\prime}}, \overrightarrow{s_{r}^{\prime}}\right)},
$$

where $\overrightarrow{s_{l}^{\prime}}\left(\overrightarrow{s_{r}^{\prime}}\right)$ is some specific configuration for the postselection. Now consider another classical post-selection probability distribution,

$$
P\left(x_{l}, x_{r}\right)=\frac{P\left(x_{l}, x_{r}, \overrightarrow{s_{l}^{\prime}}, \overrightarrow{s_{r}^{\prime}}\right)}{P\left(\overrightarrow{s_{l}^{\prime}}, \overrightarrow{s_{r}^{\prime}}\right)} .
$$


Using Eq. (A2), we can get

$$
\frac{1}{\delta^{2}} q\left(x_{l}, x_{r}\right) \leqslant P\left(x_{l}, x_{r}\right) \leqslant \delta^{2} q\left(x_{l}, x_{r}\right) .
$$

As we can see here, because $\delta<\sqrt{2}$, we can classically simulate the post-DQFNN by post-BPP, which will lead to the following containment relationship:

$$
\mathrm{PH} \subseteq \mathrm{P}^{\mathrm{PP}}=\mathrm{P}^{\text {post-BQP }}=\mathrm{P}^{\text {post-DQFNN }} \subseteq \mathrm{P}^{\text {post-BPP }} \subseteq \mathrm{p}^{\mathrm{NP}},
$$

and $\mathrm{PH} \subseteq \mathrm{p}^{\mathrm{NP}}$. So the $\mathrm{PH}$ collapses to the third level. Although this conjuncture is not as strong as $\mathrm{P}=\mathrm{NP}$, it is still generally believed that would not happen.

\section{APPENDIX B: CLASSICAL-QUANTUM HYBRID UPDATING SCHEME}

We first introduce a classical-quantum hybrid Monte Carlo sampling method which can approximate the mean value of am operator efficiently. Like the classical Monte Carlo method, the statistical error decays with the sampling size $N_{s}$ as $\frac{1}{\sqrt{N_{s}}}$ [69]. For a general Hermitian operator $\mathcal{A}$, its expectation value can be obtained by sampling a Monte Carlo chain with the probability $\rho_{l, l}=\langle l|\rho| l\rangle[36]$ :

$$
\langle\mathcal{A}\rangle=\sum_{l} \rho_{l, l} \frac{(\mathcal{A} \rho)_{l, l}}{\rho_{l, l}}=\sum_{l} \rho_{l, l} \sum_{m} \frac{\mathcal{A}_{l, m} \rho_{l, m}}{\rho_{l, l}} .
$$

Thus, $(\mathcal{A} \rho)_{l, l}$ can be computed efficiently if the variable $\mathcal{A}$ consists of only local operators.

As for the non-Hermitian operators, taking $S$ and $\vec{f}$ as an example, we first rewrite $S$ and $\vec{f}$ in an explicit form:

$$
\begin{aligned}
& f_{\mu}=\operatorname{Re}\left[\sum_{\vec{l}, \vec{r}}\left|\rho_{\vec{l}, \vec{r}}\right|^{2}\left(\frac{d \ln \left(\rho_{\vec{l}, \vec{r}}\right)}{d \Theta_{\mu}}\right)^{*} \frac{(\mathcal{L} \rho)_{\vec{l}, \vec{r}}}{\rho_{\vec{l}, \vec{r}}}\right] \\
&- \operatorname{Re}\left[\sum_{\vec{l}, \vec{r}}\left|\rho_{\vec{l}, \vec{r}}\right|^{2}\left(\frac{d \ln \left(\rho_{\vec{l}, \vec{r}}\right)}{d \Theta_{\mu}}\right)^{*} \sum_{\vec{l}^{\prime}, \vec{r}^{\prime}}\left|\rho_{\vec{l}^{\prime}, \vec{r}^{\prime}}\right|^{2} \frac{(\mathcal{L} \rho)_{\overrightarrow{l^{\prime}}, \vec{r}^{\prime}}}{\rho_{\vec{l}^{\prime}, \vec{r}^{\prime}}}\right], \\
& S_{\mu, \mu^{\prime}}=\operatorname{Re}\left[\sum_{\vec{l}, \vec{r}}\left|\rho_{\vec{l}, \vec{r}}\right|^{2}\left(\frac{d \ln \left(\rho_{\vec{l}, \vec{r}}\right)}{d \Theta_{\mu}}\right)^{*}\left(\frac{d \ln \left(\rho_{\vec{l}, \vec{r}}\right)}{d \Theta_{\mu}^{\prime}}\right)\right] \\
&-\operatorname{Re}\left[\sum_{\vec{l}, \vec{r}}\left|\rho_{\vec{l}, \vec{r}}\right|^{2}\left(\frac{d \ln \left(\rho_{\vec{l}, \vec{r}}\right)}{d \Theta_{\mu}}\right)^{*} \sum_{\vec{l}^{\prime}, \vec{r}^{\prime}}\left|\rho_{\overrightarrow{l^{\prime}}, \overrightarrow{r^{\prime}}}\right|^{2} \frac{d \ln \left(\rho_{\overrightarrow{l^{\prime}}, \overrightarrow{r^{\prime}}}\right)}{d \Theta_{\mu^{\prime}}}\right],
\end{aligned}
$$

where the right state $\vec{r}=\left(r_{0}, r_{1}, \ldots, r_{N}\right)$ and the left state $\vec{l}=$ $\left(l_{0}, l_{1}, \ldots, l_{N}\right)$ indicate a vector form of the $N$-particle state and we have $r(l)_{j}= \pm 1$ here. Then, it is easy to see that we

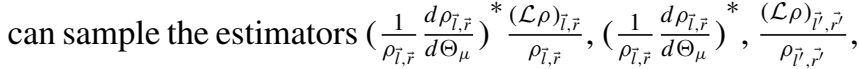

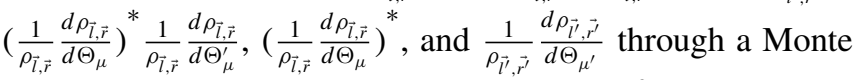
Carlo chain generated by the probability $\left|\rho_{\vec{l}, \vec{r}}\right|^{2}$.

To compute these estimators, we only need to consider three quantities: $\rho_{\vec{l}, \vec{r}}, \frac{d \rho_{\vec{l}, \vec{r}}}{d \Theta_{\mu}}$, and $(\mathcal{L} \rho)_{\vec{l}, \vec{r}}=\langle\vec{l}|\mathcal{L} \rho| \vec{r}\rangle$. The first two quantities can be measured in our DQFNN. As for the term $(\mathcal{L} \rho)_{\vec{l}, \vec{r}}=\langle\vec{l}|\mathcal{L} \rho| \vec{r}\rangle$, notice that our Lindblad equation only contains the local operators $\sigma_{j}^{z} \sigma_{j+1}^{z}, \sigma_{j}^{z} \sigma_{j+2}^{z}, \sigma_{j}^{x}, \sigma_{j}^{+,-}$, and $\sigma_{j}^{+} \sigma_{j}^{-}$, which satisfy the following relations:

$$
\begin{aligned}
& \left\langle\vec{l}\left|\sigma_{j}^{x}\right| \vec{r}\right\rangle=\delta_{r_{j},-l_{j}} \prod_{k \neq j} \delta_{r_{k}, l_{k}}, \\
& \left\langle\vec{l}\left|\sigma_{j}^{z} \sigma_{j+1}^{z}\right| \vec{r}\right\rangle=r_{j} r_{j+1} \delta_{\vec{l}, \vec{r}}, \\
& \left\langle\vec{l}\left|\sigma_{j}^{z} \sigma_{j+2}^{z}\right| \vec{r}\right\rangle=r_{j} r_{j+2} \delta_{\vec{l}, \vec{r}}, \\
& \left\langle\vec{l}\left|\sigma_{j}^{+} \sigma_{j}^{-}\right| \vec{r}\right\rangle=\delta_{r_{j}, 1} \delta_{\vec{l}, \vec{r}}, \\
& \left\langle\vec{l}\left|\sigma_{j}^{-}\right| \vec{r}\right\rangle=\delta_{r_{j}, 1} \delta_{l_{j},-1} \prod_{k \neq j} \delta_{l_{k}, r_{k}}, \\
& \left\langle\vec{l}\left|\sigma_{j}^{+}\right| \vec{r}\right\rangle=\delta_{l_{j},-1} \delta_{r_{j}, 1} \prod_{k \neq j} \delta_{l_{k}, r_{k}} .
\end{aligned}
$$

Using these relations we can obtain the term $(\mathcal{L} \rho)_{\vec{l}, \vec{r}}=$ $\langle\vec{l}|\mathcal{L} \rho| \vec{r}\rangle$

Here we only consider the one-dimensional case corresponding to Eq. $(10)$ in the main text. $(\mathcal{L} \rho)_{\vec{l}, \vec{r}}$ can be written as

$$
\begin{aligned}
\frac{(\mathcal{L} \rho)_{\vec{l}, \vec{r}}}{\rho_{\vec{l}, \vec{r}}} & -i \frac{\langle\vec{l}|[H, \rho]| \vec{r}\rangle}{\rho_{\vec{l}, \vec{r}}} \\
= & -\frac{\gamma}{2} \sum_{j}\left[\frac{\left\langle\vec{l}\left|\left\{\sigma_{j}^{+} \sigma_{j}^{-}, \rho\right\}\right| \vec{r}\right\rangle}{\rho_{\vec{l}, \vec{r}}}-2 \frac{\left\langle\vec{l}\left|\sigma_{j}^{-} \rho \sigma_{j}^{+}\right| \vec{r}\right\rangle}{\rho_{\vec{l}, \vec{r}}}\right] \\
= & \sum_{j=0}^{N}\left\{-i J\left(l_{j} l_{j+1}-r_{j} r_{j+1}\right)\right. \\
& -i h\left[\frac{\rho\left(\ldots,-l_{j}, \ldots\right)}{\rho\left(\ldots, l_{j}, \ldots\right)}-\frac{\rho\left(\ldots,-r_{j}, \ldots\right)}{\rho\left(\ldots, r_{j}, \ldots\right)}\right] \\
& \left.-\frac{\gamma}{2}\left(\delta_{l_{j}, 1}+\delta_{r_{j}, 1}\right)\right\}-\gamma \delta_{l_{j},-1} \delta_{r_{j},-1} \\
& \times \frac{\rho\left(\ldots, l_{j-1}, 1, l_{j+1}, \ldots, \ldots, r_{j-1}, 1, r_{j+1}, \ldots\right)}{\rho\left(\ldots, l_{j-1},-1, l_{j+1}, \ldots, \ldots, r_{j-1},-1, r_{j+1}, \ldots\right)} .
\end{aligned}
$$

To sample $S$ and $\vec{f}$ efficiently, we generate a Markov chain of the right and left state configurations from step zero $\left(\vec{r}_{0}, \vec{l}_{0}\right)$ to step $s\left(\vec{r}_{s}, \vec{l}_{s}\right)$. In each step, new trial right and left state configurations are randomly generated, and the new configuration

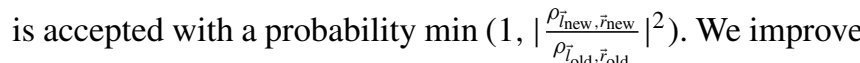
our sampling efficiency by generating each new configuration, slightly modifying the strategies discussed in [36,38]: 
Generating new configuration for $\vec{l}$ and $\vec{r}$.

Movement of spin configuration

Probability

1. For arbitrary $i$ th site, $r_{i} \rightarrow-r_{i}$ and $l_{i} \rightarrow-l_{i}$

0.33

2. For arbitrary th site, $r_{i} \rightarrow-r_{i}$ or $l_{i} \rightarrow-l_{i}$

0.33

3. For arbitrary $i$ th site,

$r_{i} \rightarrow-r_{i}, r_{i+1} \rightarrow-r_{i+1}$ or $l_{i} \rightarrow-l_{i}$

$l_{i+1} \rightarrow-l_{i+1}$

4. $\vec{r} \rightarrow-\vec{r}$ and $\vec{l} \rightarrow-\vec{l}$

0.075

5. A new configuration randomly generated

0.025

To avoid the movement falling into some specific configurations, and to approximate the expectation value of a general hermitian observable, we consider the accepted probability as $\min \left(1, \frac{\rho_{\vec{l}_{\text {new }}, \vec{l}_{\text {new }}}}{\rho_{\vec{l}_{\text {old }}, \vec{l}_{\text {old }}}}\right)$, and generate a list of state configuration $\vec{l}_{0} \rightarrow \cdots \rightarrow \vec{l}_{s}$ by the following possible moves:

Generating new configuration for $\vec{l}$.

\begin{tabular}{lc}
\hline \hline Movement of spin configuration & Probability \\
\hline 1. For arbitrary $i$ th site, $l_{i} \rightarrow-l_{i}$ & 0.49 \\
2. For arbitrary th site, $l_{i} \rightarrow-l_{i}$ and $l_{i+1} \rightarrow-l_{i+1}$ & 0.49 \\
3. A new configuration randomly generated & 0.02 \\
\hline \hline
\end{tabular}

\section{APPENDIX C: QUANTUM UPDATING SCHEME}

With quantum devices, $S$ and $\vec{f}$ can be obtained efficiently from linear combinations of measurements of proper observables in the experiment. A straightforward way is to do tomography of the output density matrix, which, however, is exponentially expensive as the system size increases. Thus, this method is limited to small systems. To overcome this difficulty, we use a swap test based method to measure $S$ and $\vec{f}$ efficiently. In this algorithm, it involves a $2 \times n$-qubit controlled-swap gate, which is defined as

$$
U_{\mathrm{C}-\mathrm{SWAP}}|x\rangle|\psi\rangle_{A}|\phi\rangle_{B}= \begin{cases}|x\rangle|\psi\rangle_{A}|\phi\rangle_{B} & \text { for } x=0, \\ |x\rangle|\phi\rangle_{A}|\psi\rangle_{B} & \text { for } x=1,\end{cases}
$$

The controlled-swap gate can be decomposed into a series of Fredkin gates, where the depth of such a quantum circuit grows $O(n)$ with respect to the output qubits number $n$. We rewrite $S$ and $\vec{f}$ as follows:

$$
\begin{aligned}
f_{\mu}= & \operatorname{Re}\left(\sum_{\vec{l}}\left\langle\vec{l}\left|\frac{\partial \rho}{\partial \Theta_{\mu}} \mathcal{L} \rho\right| \vec{l}\right\rangle-\sum_{\vec{l}}\left\langle\vec{l}\left|\rho \frac{\partial \rho}{\partial \Theta_{\mu}}\right| \vec{l}\right\rangle\right. \\
& \left.\times \sum_{\vec{l}^{\prime}}\left\langle\vec{l}^{\prime}|\rho \mathcal{L} \rho| \vec{l}^{\prime}\right\rangle\right), \\
S_{\mu, \nu}= & \operatorname{Re}\left(\sum_{\vec{l}}\left\langle\vec{l}\left|\frac{\partial \rho}{\partial \Theta_{\mu}} \frac{\partial \rho}{\partial \Theta_{\nu}}\right| \vec{l}\right\rangle-\sum_{\vec{l}}\left\langle\vec{l}\left|\frac{\partial \rho}{\partial \Theta_{\mu}} \rho\right| \vec{l}\right\rangle\right. \\
& \left.\times \sum_{\vec{l}^{\prime}}\left\langle\vec{l}^{\prime}\left|\frac{\partial \rho}{\partial \Theta_{\nu}} \rho\right| \vec{l}^{\prime}\right\rangle\right) .
\end{aligned}
$$

Thanks to the special structures of DQFNNs studied in this paper, the derivative $\frac{\partial \rho}{\partial \Theta_{\mu}}$ of $\rho$ with respect to the parameter $\Theta_{\mu}$ can be written as Eq. (10) in the main text. For the convenience, we write $\sum_{\vec{l}}\langle\vec{l}|\cdots| \vec{l}\rangle$ as a trace operation, and Eqs. (C2) and (C3) then reduce to

$$
\begin{aligned}
f_{\mu}=\frac{1}{2} \operatorname{Re}\left\{\operatorname{Tr}\left[\rho\left(\Theta_{\mu}+\frac{\pi}{2}\right) \mathcal{L} \rho\right]-\operatorname{Tr}\left[\rho\left(\Theta_{\mu}-\frac{\pi}{2}\right) \mathcal{L} \rho\right]-\left\{\operatorname{Tr}\left[\rho\left(\Theta_{\mu}+\frac{\pi}{2}\right) \rho\right]-\operatorname{Tr}\left[\rho\left(\Theta_{\mu}-\frac{\pi}{2}\right) \rho\right]\right\}[\operatorname{Tr}(\rho \mathcal{L} \rho)]\right\}, \\
S_{\mu, \nu}=\frac{1}{2} \operatorname{Re}\left\{\operatorname{Tr}\left[\rho\left(\Theta_{\mu}+\frac{\pi}{2}\right) \rho\left(\Theta_{\nu}+\frac{\pi}{2}\right)\right]+\operatorname{Tr}\left[\rho\left(\Theta_{\mu}-\frac{\pi}{2}\right) \rho\left(\Theta_{v}+\frac{\pi}{2}\right)\right]\right\} \\
+\frac{1}{2} \operatorname{Re}\left\{\operatorname{Tr}\left[\rho\left(\Theta_{\mu}+\frac{\pi}{2}\right) \rho\left(\Theta_{v}-\frac{\pi}{2}\right)\right]+\operatorname{Tr}\left[\rho\left(\Theta_{\mu}-\frac{\pi}{2}\right) \rho\left(\Theta_{v}-\frac{\pi}{2}\right)\right]\right\} \\
-\frac{1}{2} \operatorname{Re}\left\{\left\{\operatorname{Tr}\left[\rho\left(\Theta_{\mu}+\frac{\pi}{2}\right) \rho\right]-\operatorname{Tr}\left[\rho\left(\Theta_{\mu}-\frac{\pi}{2}\right) \rho\right]\right\}\left\{\operatorname{Tr}\left[\rho\left(\Theta_{\nu}+\frac{\pi}{2}\right) \rho\right]-\operatorname{Tr}\left[\rho\left(\Theta_{v}-\frac{\pi}{2}\right) \rho\right]\right\}\right\} .
\end{aligned}
$$

As we can see in Eqs. (C4)-(C5), each term in $f_{\mu}$ and $S_{\mu, v}$ only has two general forms, namely, $\operatorname{Tr}\left[\rho(\Theta) \rho\left(\Theta^{\prime}\right)\right]$ and $\operatorname{Tr}\left[\rho(\Theta) \mathcal{L} \rho\left(\Theta^{\prime}\right)\right]$, where $\Theta$ and $\Theta^{\prime}$ are two parameter sets that can be different or identical.

We adopt the quantum circuit in Fig. 7(a) to measure $\operatorname{Tr}\left[\rho(\Theta) \rho\left(\Theta^{\prime}\right)\right]$, which has been discussed in [70]. We first generate the output density matrices $\rho(\Theta)$ and $\rho\left(\Theta^{\prime}\right)$ from two DQFNNs, and introduce another ancillary qubit which is initialized to the $(|0\rangle+|1\rangle) / \sqrt{2}$ state. We decompose the spectrum of the whole system: $\rho_{w}=\frac{1}{2}(|0\rangle\langle 0|+| 0\rangle\langle 1|+| 1\rangle\langle 0|+| 1\rangle\langle 1|) \otimes$ $\sum_{\vec{l}, \vec{r}, \vec{l}^{\prime}, \vec{r}^{\prime}} \rho_{\vec{l}, \vec{r}} \rho_{\vec{l}^{\prime}, \vec{r}^{\prime}}^{\prime}\left|\vec{l}, \vec{l}^{\prime}\right\rangle\left\langle\vec{r}, \vec{r}^{\prime}\right|$.

After that, we apply a controlled-swap gate on the whole system and obtain

$$
\begin{aligned}
U_{\mathrm{C}-\mathrm{SWAP}} \rho_{w} U_{\mathrm{C}-\mathrm{SWAP}}^{\dagger}= & \frac{1}{2}|0\rangle\left\langle 0\left|\otimes \sum_{\vec{l}, \vec{r}, \vec{l}^{\prime}, \vec{r}^{\prime}} \rho_{\vec{l}, \vec{r}} \rho_{\vec{l}^{\prime}, \vec{r}^{\prime}}^{\prime}\right| \vec{l}, \vec{l}^{\prime}\right\rangle\left\langle\vec{r}, \vec{r}^{\prime}\left|+\frac{1}{2}\right| 0\right\rangle\left\langle 1\left|\otimes \sum_{\vec{l}, \vec{r}, \vec{l}^{\prime}, \vec{r}^{\prime}} \rho_{\vec{l}, \vec{r}} \rho_{\vec{l}^{\prime}, \vec{r}^{\prime}}^{\prime}\right| \vec{l}, \vec{l}^{\prime}\right\rangle\left\langle\vec{r}^{\prime}, \vec{r}\right| \\
& +\frac{1}{2}|1\rangle\left\langle 0\left|\otimes \sum_{\vec{l}, \vec{r}, \vec{l}^{\prime}, \vec{r}^{\prime}} \rho_{\vec{l}, \vec{r}} \rho_{\vec{l}^{\prime}, \vec{r}^{\prime}}^{\prime}\right| \vec{l}^{\prime}, \vec{l}\right\rangle\left\langle\vec{r}, \vec{r}^{\prime}\left|+\frac{1}{2}\right| 1\right\rangle\left\langle 1\left|\otimes \sum_{\vec{l}, \vec{r}, \vec{l}^{\prime}, \vec{r}^{\prime}} \rho_{\vec{l}, \vec{r}} \rho_{\vec{l}^{\prime}, \vec{r}^{\prime}}^{\prime}\right| \vec{l}^{\prime}, \vec{l}\right\rangle\left\langle\vec{r}^{\prime}, \vec{r}\right| .
\end{aligned}
$$




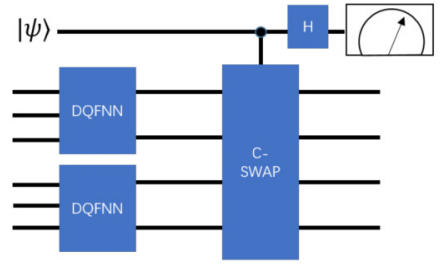

(a)

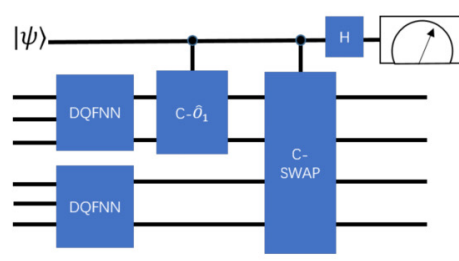

(b)

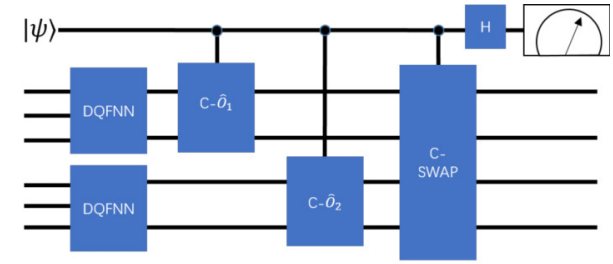

(c)

FIG. 7. The quantum circuits for obtaining $S$ and $\vec{f}$ used in the DQFNN approach. Here, H denotes the Hadamard gate and C-SWAP represents the controlled-swap gate defined in Eq. (C1). Each circuit is composed of two DQFNNs to generate $\rho(\Theta)$ and $\rho\left(\Theta^{\prime}\right)$ and one ancillary qubit for measuring. (a) The circuit for measuring $\operatorname{Re}\left\{\operatorname{Tr}\left[\rho(\Theta) \rho\left(\Theta^{\prime}\right)\right]\right\}$, where $|\psi\rangle=(|0\rangle+|1\rangle) / \sqrt{2}$. (b) The circuit for measuring $\operatorname{Tr}\left[\rho(\Theta) \hat{O}_{1} \rho\left(\Theta^{\prime}\right)\right]$. C- $\hat{O}_{1}$ is the controlled- $\hat{O}_{1}$ gate defined in Eq. (C9), where $|\psi\rangle=(|0\rangle+|1\rangle) / \sqrt{2}$ for measuring the real part of the quantity and $|\psi\rangle=(|0\rangle+i|1\rangle) / \sqrt{2}$ for measuring the imaginary part of the quantity. (c) The circuit for measuring $\operatorname{Tr}\left[\rho(\Theta) \hat{O}_{1} \rho\left(\Theta^{\prime}\right) \hat{O}_{2}\right]$, where $|\psi\rangle$ is initialized by the same strategy as in (b).

Finally, we apply another Hadamard gate on the ancilla qubit and measure the spin probability in state $|0\rangle$ :

$$
P(0)=\frac{1}{2}+\frac{1}{2} \operatorname{Tr}\left[\rho(\Theta) \rho\left(\Theta^{\prime}\right)\right],
$$

where $P(0)$ is the probability that the ancillary qubit is in state $|0\rangle$, so that we can obtain $\operatorname{Tr}\left[\rho(\Theta) \rho\left(\Theta^{\prime}\right)\right]$ via measuring the ancillary state population.

To measuring $\operatorname{Tr}\left[\rho(\Theta) \mathcal{L} \rho\left(\Theta^{\prime}\right)\right]$, we expand the Lindblad equation into a explicit form:

$$
\begin{aligned}
\operatorname{Tr}\left[\rho(\Theta) \mathcal{L} \rho\left(\Theta^{\prime}\right)\right]= & -i \operatorname{Tr}\left[\rho(\Theta) H \rho\left(\Theta^{\prime}\right)\right]+i \operatorname{Tr}\left[\rho\left(\Theta^{\prime}\right) H \rho(\Theta)\right] \\
& -\frac{\gamma}{2} \sum_{i}\left\{\operatorname{Tr}\left[\rho(\Theta) \sigma_{i}^{+} \sigma_{i}^{-} \rho\left(\Theta^{\prime}\right)\right]+\operatorname{Tr}\left[\rho\left(\Theta^{\prime}\right) \sigma_{i}^{+} \sigma_{i}^{-} \rho(\Theta)\right]-2 \operatorname{Tr}\left[\sigma_{i}^{-} \rho(\Theta) \sigma_{i}^{+} \rho\left(\Theta^{\prime}\right)\right]\right\} .
\end{aligned}
$$

Taking into account the one-dimensional case [see Eq. (10) in main text], the Hamiltonian $H$ only involves the local Pauli operators, so that we only need to consider $\operatorname{Tr}\left[\rho(\Theta) \sigma_{i}^{x} \rho\left(\Theta^{\prime}\right)\right]$ and $\operatorname{Tr}\left[\rho(\Theta) \sigma_{i}^{z} \sigma_{i+1}^{z} \rho\left(\Theta^{\prime}\right)\right]$. Here, we write the local Pauli operators in a uniform form $\hat{O}=\sigma_{i}^{x}, \sigma_{i}^{z} \sigma_{i+1}^{z}$. Utilizing the quantum circuits in Fig. 7(b), we can measure the real and imaginary parts of $\operatorname{Tr}\left\{\rho(\Theta) \hat{O} \rho\left(\Theta^{\prime}\right)\right\}$ separately by setting the initial state of the ancillary qubit as $(|0\rangle+|1\rangle) / \sqrt{2}$ for measuring the real part and $(|0\rangle+i|1\rangle) / \sqrt{2}$ for measuring the imaginary part. Then, we introduce a controlled- $\hat{O}$ gate, which is defined as

$$
\hat{O}^{c}|x\rangle|\psi\rangle=|x\rangle \hat{O}^{x}|\psi\rangle \text {. }
$$

We apply the controlled-O $\hat{O}$ gate between the first subsystem and the ancillary qubit and apply another controlled-swap gate as

$$
\begin{aligned}
U_{\mathrm{C}-\mathrm{SWAP}} \hat{O}^{c} \rho_{w}^{\mathrm{Re}}\left(\hat{O}^{c}\right)^{\dagger} U_{\mathrm{C}-\mathrm{SWAP}}^{\dagger}= & \frac{1}{2}|0\rangle\left\langle 0\left|\otimes \sum_{\vec{l}, \vec{r}, \vec{l}^{\prime}, \vec{r}^{\prime}} \rho_{\vec{l}, \vec{r}} \rho_{\vec{l}^{\prime}, \vec{r}^{\prime}}^{\prime}\right| \vec{l}, \vec{l}^{\prime}\right\rangle\left\langle\vec{r}, \vec{r}^{\prime}\left|+\frac{1}{2}\right| 0\right\rangle\left\langle 1\left|\otimes \sum_{\vec{l}, \vec{r}, \vec{l}^{\prime}, \vec{r}^{\prime}} \rho_{\vec{l}, \vec{r}} \rho_{\vec{l}^{\prime}, \vec{r}^{\prime}}^{\prime}\right| \vec{l}, \vec{l}^{\prime}\right\rangle\left\langle\vec{r}^{\prime}, \vec{r}\right| \hat{O}^{\dagger} \\
& +\frac{1}{2}|1\rangle\left\langle 0\left|\otimes \sum_{\vec{l}, \vec{r}, \vec{l}^{\prime}, \vec{r}^{\prime}} \rho_{\vec{l}, \vec{r}} \rho_{\vec{l}^{\prime}, \vec{r}^{\prime}}^{\prime} \hat{O}\right| \vec{l}^{\prime}, \vec{l}\right\rangle\left\langle\vec{r}, \vec{r}^{\prime}\left|+\frac{1}{2}\right| 1\right\rangle\left\langle 1\left|\otimes \sum_{\vec{l}, \vec{r}, \vec{l}^{\prime}, \vec{r}^{\prime}} \rho_{\vec{l}, \vec{r}} \rho_{\vec{l}^{\prime}, \vec{r}^{\prime}}^{\prime} \hat{O}\right| \vec{l}^{\prime}, \vec{l}\right\rangle\left\langle\vec{r}^{\prime}, \vec{r}\right| \hat{O}^{\dagger}
\end{aligned}
$$

and

$$
\begin{aligned}
U_{\mathrm{C}-\mathrm{SWAP}} \hat{O}^{c} \rho_{w}^{\mathrm{Im}}\left(\hat{O}^{c}\right)^{\dagger} U_{\mathrm{C}-\mathrm{SWAP}}^{\dagger}= & \frac{1}{2}|0\rangle\left\langle 0\left|\otimes \sum_{\vec{l}, \vec{r}, \overrightarrow{l^{\prime}}, \vec{r}^{\prime}} \rho_{\vec{l}, \vec{r}} \rho_{\vec{l}^{\prime}, \vec{r}^{\prime}}^{\prime}\right| \vec{l}, \vec{l}^{\prime}\right\rangle\left\langle\vec{r}, \vec{r}^{\prime}\left|-\frac{i}{2}\right| 0\right\rangle\left\langle 1\left|\otimes \sum_{\vec{l}, \vec{r}, \overrightarrow{l^{\prime}}, \overrightarrow{r^{\prime}}} \rho_{\vec{l}, \vec{r}} \rho_{\vec{l}^{\prime}, \vec{r}^{\prime}}^{\prime}\right| \vec{l}, \vec{l}^{\prime}\right\rangle\left\langle\vec{r}^{\prime}, \vec{r}\right| \hat{O}^{\dagger} \\
& +\frac{i}{2}|1\rangle\left\langle 0\left|\otimes \sum_{\vec{l}, \vec{r}, \vec{l}^{\prime}, \vec{r}^{\prime}} \rho_{\vec{l}, \vec{r}} \rho_{\vec{l}^{\prime}, \vec{r}^{\prime}}^{\prime} \hat{O}\right| \vec{l}^{\prime}, \vec{l}\right\rangle\left\langle\vec{r}, \vec{r}^{\prime}\left|+\frac{1}{2}\right| 1\right\rangle\left\langle 1\left|\otimes \sum_{\vec{l}, \vec{r}, \vec{l}^{\prime}, \vec{r}^{\prime}} \rho_{\vec{l}, \vec{r}} \rho_{\vec{l}^{\prime}, \vec{r}^{\prime}}^{\prime} \hat{O}\right| \vec{l}^{\prime}, \vec{l}\right\rangle\left\langle\vec{r}^{\prime}, \vec{r}\right| \hat{O}^{\dagger} .
\end{aligned}
$$

After applying a Hadamard gate on the ancillary qubit, we measure the spin population of the ancillary qubit,

$$
P(0)_{\operatorname{Re}}=\frac{1}{2}+\frac{1}{2} \operatorname{Re}\left\{\operatorname{Tr}\left[\rho(\Theta) \hat{O} \rho\left(\Theta^{\prime}\right)\right]\right\},
$$

and

$$
P(0)_{\operatorname{Im}}=\frac{1}{2}-\frac{1}{2} \operatorname{Im}\left\{\operatorname{Tr}\left[\rho(\Theta) \hat{O} \rho\left(\Theta^{\prime}\right)\right]\right\},
$$

and obtain $\operatorname{Tr}\left[\rho(\Theta) \hat{O} \rho\left(\Theta^{\prime}\right)\right]=2 P(0)_{\operatorname{Re}}-1+i\left[1-2 P(0)_{\operatorname{Im}}\right]$.

To calculate the term $\operatorname{Tr}\left[\rho(\Theta) \sigma_{i}^{+} \sigma_{i}^{-} \rho\left(\Theta^{\prime}\right)\right]$, we divide it into $\operatorname{Tr}\left[\rho(\Theta) \rho\left(\Theta^{\prime}\right)\right]$ and $\operatorname{Tr}\left[\rho(\Theta) \sigma_{i}^{z} \rho\left(\Theta^{\prime}\right)\right]$ by using ${\sigma_{i}^{+}}_{i}^{-}=\frac{1}{2}+$ $\frac{1}{2} \sigma_{i}^{z}$. These two quantities can be measured by using the method above. We expand the last term $\sigma_{i}^{-} \rho \sigma_{i}^{+}=\sigma_{i}^{x} \rho \sigma_{i}^{x}+\sigma_{i}^{y} \rho \sigma_{i}^{y}-$ 
$i\left(\sigma_{i}^{y} \rho \sigma_{i}^{x}-\sigma_{i}^{x} \rho \sigma_{i}^{y}\right)$ and measure the real part and imaginary part separately. To calculate $\operatorname{Tr}\left[\rho(\Theta) \sigma_{i}^{x, y} \rho\left(\Theta^{\prime}\right) \sigma_{i}^{x, y}\right]$, as shown in Fig. 7(c), we apply two controlled- $\hat{O}_{1,2}$ gates, where $\hat{O}_{1,2}=\sigma_{i}^{x, y}$, and apply the controlled-swap gate,

$$
\begin{aligned}
U_{\mathrm{C}-\mathrm{SWAP}} \hat{O}_{2}^{c} \hat{O}_{1}^{c} \rho_{w}^{R e}\left(\hat{O}_{1}^{c}\right)^{\dagger}\left(\hat{O}_{2}^{c}\right)^{\dagger} U_{\mathrm{C}-\mathrm{SWAP}}^{\dagger}= & \frac{1}{2}|0\rangle\left\langle 0 \mid \otimes \sum_{\vec{l}, \vec{r}, \vec{l}^{\prime}, \vec{r}^{\prime}} \rho_{\vec{l}, \vec{r}} \rho_{\vec{l}^{\prime}, \vec{r}^{\prime}}^{\prime}, \vec{l}, \vec{l}^{\prime}\right\rangle\left\langle\vec{r}, \vec{r}^{\prime}\left|+\frac{1}{2}\right| 0\right\rangle\left\langle 1 \mid \otimes \sum_{\vec{l}, \vec{r}, \vec{l}^{\prime}, \vec{r}^{\prime}} \rho_{\vec{l}, \vec{r}} \rho_{\vec{l}^{\prime}, \vec{r}^{\prime}}^{\prime} \vec{l}, \vec{l}^{\prime}\right\rangle\left\langle\vec{r}^{\prime}, \vec{r}\right| \hat{O}_{1}^{\dagger} \hat{O}_{2}^{\dagger} \\
& +\frac{1}{2}|1\rangle\left\langle 0\left|\otimes \sum_{\vec{l}, \vec{r}, \vec{l}^{\prime}, \vec{r}^{\prime}} \rho_{\vec{l}, \vec{r}} \rho_{\vec{l}^{\prime}, \vec{r}^{\prime}}^{\prime} \hat{O}_{1} \hat{O}_{2}\right| \vec{l}^{\prime}, \vec{l}\right\rangle\left\langle\vec{r}, \vec{r}^{\prime}\right| \\
& +\frac{1}{2}|1\rangle\left\langle 1\left|\otimes \sum_{\vec{l}, \vec{r}, \vec{l}^{\prime}, \vec{r}^{\prime}} \rho_{\vec{l}, \vec{r}} \rho_{\vec{l}^{\prime}, \vec{r}^{\prime}}^{\prime} \hat{O}_{1} \hat{O}_{2}\right| \vec{l}^{\prime}, \vec{l}\right\rangle\left\langle\vec{r}^{\prime}, \vec{r}\right| \hat{O}_{1}^{\dagger} \hat{O}_{2}^{\dagger}
\end{aligned}
$$

and

$$
\begin{aligned}
U_{\mathrm{C}-\mathrm{SWAP}} \hat{O}_{2}^{c} \hat{O}_{1}^{c} \rho_{w}^{I m}\left(\hat{O}_{1}^{c}\right)^{\dagger}\left(\hat{O}_{2}^{c}\right)^{\dagger} U_{\mathrm{C}-\mathrm{SWAP}}^{\dagger}= & \frac{1}{2}|0\rangle\left\langle 0\left|\otimes \sum_{\vec{l}, \vec{r}, \vec{l}^{\prime}, \vec{r}^{\prime}} \rho_{\vec{l}, \vec{r}} \rho_{\vec{l}^{\prime}, \vec{r}^{\prime}}^{\prime}\right| \vec{l}, \vec{l}^{\prime}\right\rangle\left\langle\vec{r}, \vec{r}^{\prime}\left|-\frac{i}{2}\right| 0\right\rangle\left\langle 1\left|\otimes \sum_{\vec{l}, \vec{r}, \vec{l}^{\prime}, \vec{r}^{\prime}} \rho_{\vec{l}, \vec{r}} \rho_{\vec{l}^{\prime}, \vec{r}^{\prime}}^{\prime}\right| \vec{l}, \vec{l}^{\prime}\right\rangle\left\langle\vec{r}^{\prime}, \vec{r}\right| \hat{O}_{1}^{\dagger} \hat{O}_{2}^{\dagger} \\
& +\frac{i}{2}|1\rangle\left\langle 0\left|\otimes \sum_{\vec{l}, \vec{r}, \vec{l}^{\prime}, \vec{r}^{\prime}} \rho_{\vec{l}, \vec{r}} \rho_{\vec{l}^{\prime}, \vec{r}^{\prime}}^{\prime} \hat{O}_{1} \hat{O}_{2}\right| \vec{l}^{\prime}, \vec{l}\right\rangle\left\langle\vec{r}, \vec{r}^{\prime}\right| \\
& +\frac{1}{2}|1\rangle\left\langle 1\left|\otimes \sum_{\vec{l}, \vec{r}, \vec{l}^{\prime}, \vec{r}^{\prime}} \rho_{\vec{l}, \vec{r}} \rho_{\vec{l}^{\prime}, \vec{r}^{\prime}}^{\prime} \hat{O}_{1} \hat{O}_{2}\right| \vec{l}^{\prime}, \vec{l}\right\rangle\left\langle\vec{r}^{\prime}, \vec{r}\right| \hat{O}_{1}^{\dagger} \hat{O}_{2}^{\dagger} .
\end{aligned}
$$

Then, similarly to our previous discussion, we measure the ancillary qubit,

$$
P(0)_{\operatorname{Re}}=\frac{1}{2}+\frac{1}{2} \operatorname{Re}\left\{\operatorname{Tr}\left[\rho(\Theta) \hat{O} \rho\left(\Theta^{\prime}\right)\right]\right\}
$$

and

$$
P(0)_{\operatorname{Im}}=\frac{1}{2}-\frac{1}{2} \operatorname{Im}\left\{\operatorname{Tr}\left[\rho(\Theta) \hat{O} \rho\left(\Theta^{\prime}\right)\right]\right\}
$$

and obtain $\operatorname{Tr}\left[\rho(\Theta) \hat{O}_{1} \rho\left(\Theta^{\prime}\right) \hat{O}_{2}\right]=2 P(0)_{\operatorname{Re}}-1+i[1-$ $\left.2 P(0)_{\text {Im }}\right]$.

It is easy to see that $\operatorname{Tr}[\rho(\Theta) \hat{O} \rho(\Theta)]$ and $\operatorname{Tr}\left[\rho(\Theta) \hat{O}_{1} \rho(\Theta) \hat{O}_{2}\right]$ are real numbers, so they can be measured by the quantum circuits in Figs. 7(b) and 7(c) respectively by setting the initial ancillary qubit state as $(|0\rangle+|1\rangle) / \sqrt{2}$. In summary, by using the techniques discussed above we can obtain $S$ and $\vec{f}$ efficiently with quantum devices.

\section{APPENDIX D: TRAINING SCHEME}

We use the following procedures to train the DQFNN for solving the dynamics and stationary states for open quantum systems:

I. DQFNN initialization: In our case, only the single-qubit gate is parametrized by $R(\vec{\theta})=e^{i \theta_{1} \sigma_{z} / 2} e^{i \theta_{2} \sigma_{x} / 2} e^{i \theta_{3} \sigma_{z} / 2}$. At the beginning, we initialize the DQFNN by assigning small random numbers to all the parameters, so that each single-qubit gate is approach an identity matrix.

II. Feed forward:

(1) Set $i=1$, and set the state of qubits in the input layer to $\rho_{o}^{i}=\left(\otimes_{k=1}^{N_{1}}|+\rangle_{k}\right)\left(\otimes_{k=1}^{N_{1}}\left\langle+\left.\right|_{k}\right)\right.$, where $N_{1}$ is the number of qubits in input layer, and $|+\rangle_{k}=(|0\rangle+$ |1) $) / \sqrt{2}$.
(2) Set the state of qubits in the $(i+1)$ th layer to $\rho_{\text {in }}^{i+1}=\left(\otimes_{k=1}^{N_{i+1}}|+\rangle_{k}\right)\left(\otimes_{k=1}^{N_{i+1}}\left\langle+\left.\right|_{k}\right)\right.$, where $N_{i+1}$ is the number of qubits in the $(i+1)$ th layer.

(3) Apply unitary operations between the $i$ th layer and the $(i+1)$ th layer,

$$
\rho^{i+1}=U_{i}^{w_{i}} \cdots U_{i}^{j} \cdots U_{i}^{1}\left(\rho_{o}^{i} \otimes \rho_{\mathrm{in}}^{i+1}\right) U_{i}^{1^{\dagger}} \cdots U_{i}^{j^{\dagger}} \cdots U_{i}^{w_{i} \dagger},
$$

where $w_{i}$ is the number of perceptrons between the $i$ th layer and the $(i+1)$ th layer.

(4) Partially trace the $i$ th layer, namely, $\rho_{o}^{i+1}=$ $\operatorname{Tr}_{i}\left(\rho^{i+1}\right)$, and set $i=i+1$.

(5) Reuse the qubits. If $i<d$, the partially traced qubits in the $i$ th layer are reinitialized as $(|0\rangle+|1\rangle) / \sqrt{2}$ and they can be used in the rest of the network.

(6) Repeat II.2-II.4 until $i=d$.

III. Update parameters:

(1) Compute $S$ and $\vec{f}$. Per the discussion in Appendixes B and C, we can obtain $S$ and $\vec{f}$ via a stochastic Markovchain sampling or directly measure these two quantities.

(2) Update parameters. According to the system equation in the main text, the parameters can be updated as $\Theta_{\mu}^{\text {new }}=\Theta_{\mu}^{\text {old }}+\lambda \sum_{\nu}\left(S^{-1}\right)_{\mu, \nu} f_{v}$. Here we choose $\lambda$ to be small enough to monitor the convergence of the optimization process.

IV. Repeat steps II and III until $\delta \mathcal{L}$ reaches the minimum and the process converges.

\section{APPENDIX E: ROBUST TO NOISES}

We numerically demonstrate the robustness of our DQFNN approach against experimental noise. Here, we take two different types of noise as examples. The first type is the measurement noise. On each measurement result $\langle l|\rho| r\rangle$, we 
(a)
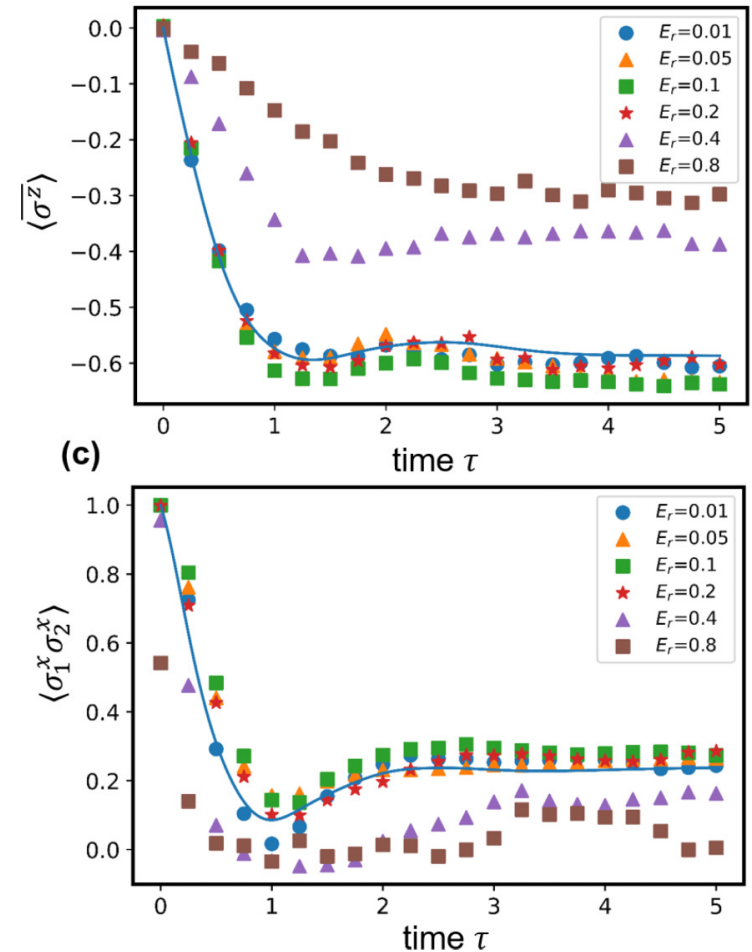

(b)
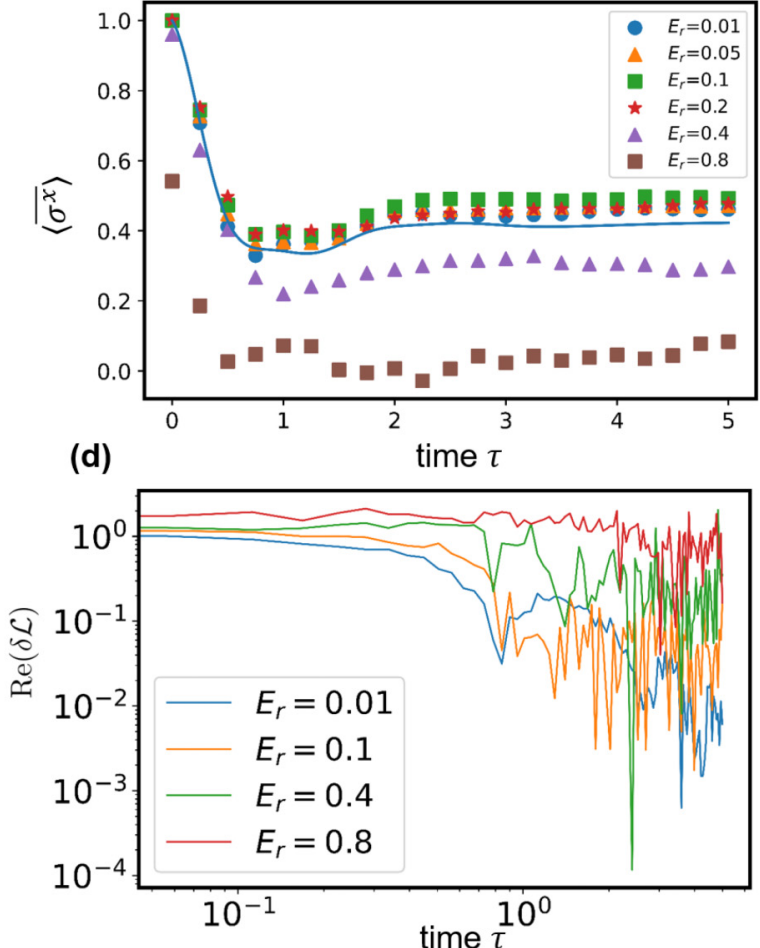

FIG. 8. Numerical demonstration of the robustness of the DQFNN against the measurement noise on solving the steady state of the transverse Ising model with transverse field $h=1.1$ and the dissipation rate $\gamma=1.0$. (a), (b), and (c) show respectively $\overline{\left\langle\sigma^{z}\right\rangle}, \overline{\left\langle\sigma^{x}\right\rangle}$, and $\left\langle\sigma_{1}^{x} \sigma_{2}^{x}\right\rangle$ as a function of evolution time. The DQFNN has the structure $(2,2,3,3,5)$ with different measurement noise levels $E_{r}=0.01,0.05,0.1,0.2,0.4,0.8$. (d) plots the real parts of $\delta \mathcal{L}$, which converge to $10^{-2}$ as the amplitudes of noise are $E_{r}=0.01$ and $E_{r}=0.1$, while they get lost for $E_{r}=0.4$ and $E_{r}=0.8$.

add the Gaussian noise, which is generated by the probability

$$
P(x)=\frac{1}{E_{r} \sqrt{2 \pi}} e^{-\frac{x^{2}}{2 E_{r}{ }^{2}}},
$$

where the $E_{r}$ denotes the standard deviation and the $x$ is the random noise variable we take.

Anothor type of noise is the decoherence noise. In our simulation, inside each perceptron, we apply a noisy quantum channel for each single-qubit state with the form

$$
\rho^{\text {out }}=\sum_{i=0}^{3} M_{i} \rho M_{i}^{\dagger},
$$

where the general operator $M_{i}$ takes the following form: $M_{0}=$ $\sqrt{1-\frac{3}{4} \epsilon \mathbb{I}}, M_{1}=\sqrt{\frac{1}{4} \epsilon} \sigma_{x}, M_{2}=\sqrt{\frac{1}{4} \epsilon} \sigma_{y}$, and $M_{3}=\sqrt{\frac{1}{4} \epsilon \sigma_{z}}$.

We first plot the effect of the measurement error on the dynamics behavior. In Figs. 8(a)-8(d), we take the DQFNN structure with $(2,2,3,3,5)$ and take the one-dimensional Ising Hamiltonian with the parameters $J=1.0$ and $h=1.1$. The dissipative rate of the Lindblad operators is $\gamma=1.0$. We plot the magnetization values $\overline{\left\langle\sigma^{x, z}\right\rangle}$ and correlations $\left\langle\sigma_{1}^{x} \sigma_{2}^{x}\right\rangle$ with different noise strengths $E_{r}=0.01,0.05,0.1,0.2,0.4,0.8$, and compare their corresponding results obtained by ED. The added noise does cause small deviations during the dynamic process in the range from low to moderate noise amplitude (smaller than 0.2 here), but the DQFNN can still converge to the steady state with good accuracy. For larger noise ampli- tude, the training process would not converge to the desirable steady state.

To demonstrate the error robustness against the two types of error in finding the steady state, we compute the fidelity between the converged output states from our DQFNN and the steady state calculated by the exact diagonalization, namely,

$$
F\left(\rho_{\mathrm{ss}}, \rho_{\mathrm{ED}}\right)=\left[\operatorname{tr} \sqrt{\sqrt{\rho_{\mathrm{ss}}} \rho_{\mathrm{ED}} \sqrt{\rho_{\mathrm{ss}}}}\right]^{2},
$$

under the measurement noise and decoherence noise with noise amplitudes $E_{r}$ and $\epsilon$. A phase-transition-like behavior is shown in both Figs. 9(a) and 9(b). This phenomenon can be interpreted as follows: when the noise is small, the effect of noise can cause a small perturbation around the real physical path, which can be diminished by the long-time evolution and will not affect the system's convergence to its steady state. However, when noise is large enough, the long-time evolution is unable to drive the system back to the original path, which decreases the output fidelity. For the measurement error, in Fig. 9(a), the threshold of the noise amplitude is around $E_{r}=0.2$, larger than the value at which the training process may fail. Considering the experimental realizations, for each single sampling, the required number of measurements scales as $O\left(\log \left(\frac{1}{\epsilon_{M}}\right)\right)$ with respect to the measurement precision $\epsilon_{M}$. Hence, to obtain a good convergence result, $\epsilon_{M}$ should be chosen much smaller than such a threshold, which corresponds to moderate times of measurement. For the decoherence error, in Fig. 9(b), the threshold of the noise amplitude is around 
(a)

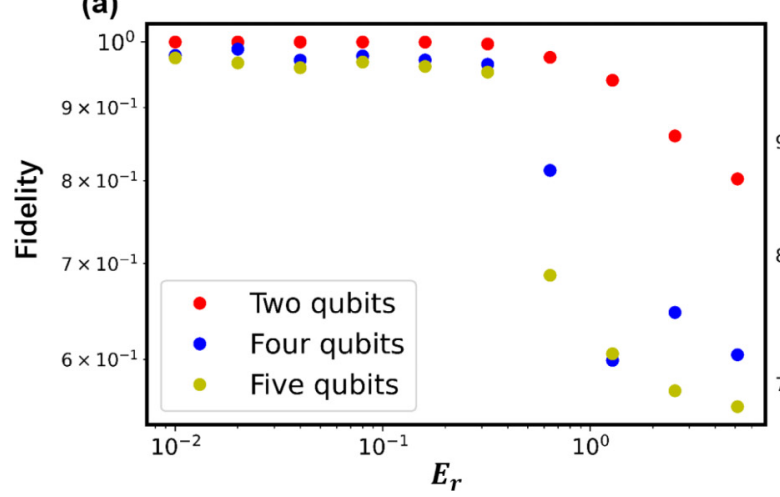

(b)

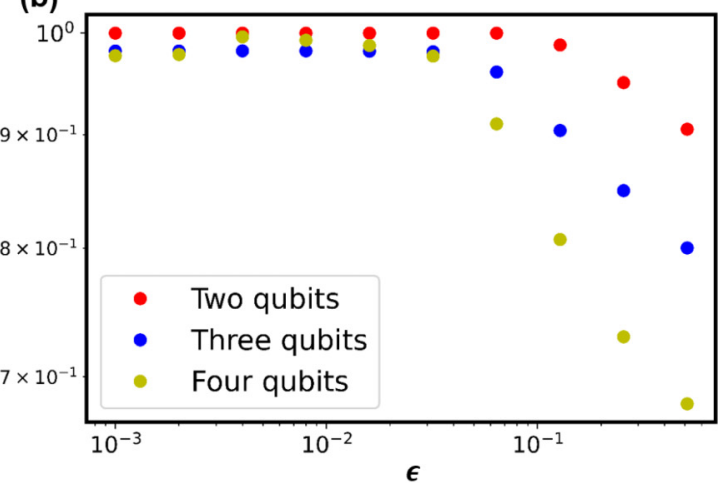

FIG. 9. Numerical demonstration of the robustness of the DQFNN against the measurement noise and decoherence noise on finding the steady state of the transverse Ising model with transverse field $h=1.1$ and the dissipation rate $\gamma=1.0$. (a) We take the measurement noise as an example. Here, we simulate three different structures of the DQFNN, namely, $(2,2,2),(2,2,2,2,4)$, and $(2,2,3,3,5)$. The noise amplitude ranges from 0.01 to 10.24 . We calculate the fidelity between the DQFNN result as converged and the physical steady state solved by ED. As it is shown in the figure, when the noise amplitude $E_{r}$ is larger than 0.2 the training process is lost and cannot find the steady-state accuracy. (b) We take the decoherence noise as an example. Here we simulate the $(2,2,2),(3,3,3)$, and $(2,2,2,2,4)$ structures of the DQFNN. The noise amplitude $\epsilon$ is ranging from 0.001 to 0.5 . Like the behavior in (a), as $\epsilon>0.04$, the fidelity decays dramatically.

$\epsilon=0.04$, which is experimentally feasible. Hence, DQFNN shows potential applications in NISQ devices.

\section{APPENDIX F: COMPUTATIONAL COMPLEXITY}

For a fully connected DQFNN, the perceptrons are composed of any possible combination of two qubits from the $i$ th layer and one qubit from the $(i+1)$ th layer, where the total number of perceptrons between two neighbor layers is $C_{n_{i}}^{2} n_{i+1}$. Hence, the total number of parameters of the DQFNN is

$$
\sum_{i=1}^{d-1} C_{n_{i}}^{2} n_{i+1} \times N_{p} \approx O\left(N_{\text {All }}^{3}\right),
$$

where $d$ is the total number of layers, $n_{i}$ denotes the number of qubits in the $i$ th layer, $N_{p}$ is the number of parameters for each perceptron, and $N_{\text {All }}$ denotes the total number of qubits in the DQFNN. Here we reduce the number of parameters by using the symmetry structure in the Hamiltonian we discussed in our main text. We restrict each perceptron to only involve local connection between $i$ th and $(i+1)$ th layers. The $j$ th qubit in the $(i+1)$ th layer is only grouped with the $\left(j \bmod n_{i}\right)$ th and $\left[(j+1) \bmod n_{i}\right]$ th qubits in the $i$ th layer into the same perceptron, where mod is the modulus operation. So that there are $n_{i+1}$ perceptrons between the $i$ th layer and $(i+1)$ th layer.
The total number of parameters is

$$
\sum_{i=1}^{d-1} n_{i+1} \times N_{p} \approx O\left(N_{\mathrm{All}}\right) .
$$

which means that the number of parameters grows linearly with the system size. This leads to the computational complexity $O\left(N_{\text {All }}^{3}\right)$ in the DQFNN approach.

\section{APPENDIX G: NUMERICAL SETUP}

Our code is written in Python and the benchmark ED algorithm is implemented by Qutip [71]. To efficiently simulate the feed-forward process of the DQFNN, the GPU package CUPY is used for the matrix multiplication.

The hyperparameters are list as follows. For the SR algorithm, we add a diagonal regulator to the $S$ matrix as $S=S+\mathcal{R} \operatorname{diag}(S)$. We fix the amplitude of the regulator $\mathcal{R}=10^{-4}$ as a constant. For Fig. 2 in main text, we choose the iteration steps as $N_{s}=2000$. The batch size is chosen to be 50000 for the case when the transverse field is less than 1.0 and 10000 when the transverse field is larger than 1.0. For Fig. 3 in the main text, we take 1000 time steps to simulate the dynamics and take one point every 50 steps to plot parts (a), (b), and (c). For Fig. 4 in the main text, we choose the learning rate as $0.01 \times 0.999^{N_{s}}$ with 1000 iteration steps. The batch size is 50000 . For Fig. 8, we choose the learning rate as $5 \times 10^{-3}$, and for Fig. 9, we choose a decaying learning rate $0.05 \times 0.999^{N_{s}}$ for finding the steady state.
[1] F. Arute, K. Arya, R. Babbush, D. Bacon, J. C. Bardin, R. Barends, R. Biswas, S. Boixo, F. G. Brandao, D. A. Buell et al., Quantum supremacy using a programmable superconducting processor, Nature (London) 574, 505 (2019).

[2] C. Song, K. Xu, H. Li, Y.-R. Zhang, X. Zhang, W. Liu, Q. Guo, Z. Wang, W. Ren, J. Hao et al., Generation of multicomponent atomic Schrödinger cat states of up to 20 qubits, Science $\mathbf{3 6 5}$, 574 (2019).

[3] P. Krantz, M. Kjaergaard, F. Yan, T. P. Orlando, S. Gustavsson, and W. D. Oliver, A quantum engineer's guide to superconducting qubits, Appl. Phys. Rev. 6, 021318 (2019). 
[4] K. Wright, K. Beck, S. Debnath, J. Amini, Y. Nam, N. Grzesiak, J.-S. Chen, N. Pisenti, M. Chmielewski, C. Collins et al., Benchmarking an 11-qubit quantum computer, Nat. Commun. 10, 1 (2019).

[5] C. D. Bruzewicz, J. Chiaverini, R. McConnell, and J. M. Sage, Trapped-ion quantum computing: Progress and challenges, Appl. Phys. Rev. 6, 021314 (2019).

[6] H. Levine, A. Keesling, G. Semeghini, A. Omran, T. T. Wang, S. Ebadi, H. Bernien, M. Greiner, V. Vuletić, H. Pichler, and M. D. Lukin, Parallel Implementation of High-Fidelity Multiqubit Gates with Neutral Atoms, Phys. Rev. Lett. 123, 170503 (2019).

[7] J. Preskill, Quantum computing in the NISQ era and beyond, Quantum 2, 79 (2018).

[8] A. W. Harrow and A. Montanaro, Quantum computational supremacy, Nature (London) 549, 203 (2017).

[9] A. Peruzzo, J. McClean, P. Shadbolt, M.-H. Yung, X.-Q. Zhou, P. J. Love, A. Aspuru-Guzik, and J. L. O'Brien, A variational eigenvalue solver on a photonic quantum processor, Nat. Commun. 5, 4213 (2014).

[10] C. Kokail, C. Maier, R. van Bijnen, T. Brydges, M. Joshi, P. Jurcevic, C. Muschik, P. Silvi, R. Blatt, C. Roos et al., Self-verifying variational quantum simulation of lattice models, Nature (London) 569, 355 (2019)

[11] J.-G. Liu, Y.-H. Zhang, Y. Wan, and L. Wang, Variational quantum eigensolver with fewer qubits, Phys. Rev. Research 1, 023025 (2019).

[12] D. Wang, O. Higgott, and S. Brierley, Accelerated Variational Quantum Eigensolver, Phys. Rev. Lett. 122, 140504 (2019).

[13] E. Farhi, J. Goldstone, and S. Gutmann, A quantum approximate optimization algorithm, arXiv:1411.4028.

[14] L. Zhou, S.-T. Wang, S. Choi, H. Pichler, and M. D. Lukin, Quantum Approximate Optimization Algorithm: Performance, Mechanism, and Implementation on Near-Term Devices, Phys. Rev. X 10, 021067 (2020).

[15] N. Moll, P. Barkoutsos, L. S. Bishop, J. M. Chow, A. Cross, D. J. Egger, S. Filipp, A. Fuhrer, J. M. Gambetta, M. Ganzhorn et al., Quantum optimization using variational algorithms on near-term quantum devices, Quantum Sci. Technol. 3, 030503 (2018).

[16] M. Schuld, A. Bocharov, K. M. Svore, and N. Wiebe, Circuitcentric quantum classifiers, Phys. Rev. A 101, 032308 (2020).

[17] V. Havlíček, A. D. Córcoles, K. Temme, A. W. Harrow, A. Kandala, J. M. Chow, and J. M. Gambetta, Supervised learning with quantum-enhanced feature spaces, Nature (London) $\mathbf{5 6 7}$, 209 (2019).

[18] I. Cong, S. Choi, and M. D. Lukin, Quantum convolutional neural networks, Nat. Phys. 15, 1273 (2019).

[19] S. Lloyd and C. Weedbrook, Quantum Generative Adversarial Learning, Phys. Rev. Lett. 121, 040502 (2018).

[20] P.-L. Dallaire-Demers and N. Killoran, Quantum generative adversarial networks, Phys. Rev. A 98, 012324 (2018).

[21] L. Hu, S.-H. Wu, W. Cai, Y. Ma, X. Mu, Y. Xu, H. Wang, Y. Song, D.-L. Deng, C.-L. Zou et al., Quantum generative adversarial learning in a superconducting quantum circuit, Sci. Adv. 5, eaav2761 (2019).

[22] K. Beer, D. Bondarenko, T. Farrelly, T. J. Osborne, R. Salzmann, D. Scheiermann, and R. Wolf, Training deep quantum neural networks, Nat. Commun. 11, 808 (2020).
[23] H.-P. Breuer, F. Petruccione et al., The Theory of Open Quantum Systems (Oxford University Press on Demand, 2002).

[24] M. Zwolak and G. Vidal, Mixed-State Dynamics in OneDimensional Quantum Lattice Systems: A Time-Dependent Superoperator Renormalization Algorithm, Phys. Rev. Lett. 93, 207205 (2004).

[25] F. Verstraete, J. J. García-Ripoll, and J. I. Cirac, Matrix Product Density Operators: Simulation of Finite-Temperature and Dissipative Systems, Phys. Rev. Lett. 93, 207204 (2004).

[26] R. Orús and G. Vidal, Infinite time-evolving block decimation algorithm beyond unitary evolution, Phys. Rev. B 78, 155117 (2008).

[27] J. Cui, J. I. Cirac, and M. C. Banuls, Variational Matrix Product Operators for the Steady State of Dissipative Quantum Systems, Phys. Rev. Lett. 114, 220601 (2015).

[28] A. H. Werner, D. Jaschke, P. Silvi, M. Kliesch, T. Calarco, J. Eisert, and S. Montangero, Positive Tensor Network Approach for Simulating Open Quantum Many-Body Systems, Phys. Rev. Lett. 116, 237201 (2016)

[29] A. A. Gangat, T. I, and Y.-J. Kao, Steady States of Infinite-Size Dissipative Quantum Chains via Imaginary Time Evolution, Phys. Rev. Lett. 119, 010501 (2017).

[30] D. Jaschke, S. Montangero, and L. D. Carr, One-dimensional many-body entangled open quantum systems with tensor network methods, Quantum Sci. Technol. 4, 013001 (2018).

[31] A. Kshetrimayum, H. Weimer, and R. Orús, A simple tensor network algorithm for two-dimensional steady states, Nat. Commun. 8, 1291 (2017).

[32] Z. Yan, L. Pollet, J. Lou, X. Wang, Y. Chen, and Z. Cai, Interacting lattice systems with quantum dissipation: A quantum Monte Carlo study, Phys. Rev. B 97, 035148 (2018).

[33] A. Nagy and V. Savona, Driven-dissipative quantum Monte Carlo method for open quantum systems, Phys. Rev. A 97, 052129 (2018).

[34] G. Carleo and M. Troyer, Solving the quantum many-body problem with artificial neural networks, Science 355, 602 (2017).

[35] L. Banchi, E. Grant, A. Rocchetto, and S. Severini, Modelling non-Markovian quantum processes with recurrent neural networks, New J. Phys. 20, 123030 (2018).

[36] M. J. Hartmann and G. Carleo, Neural-Network Approach to Dissipative Quantum Many-Body Dynamics, Phys. Rev. Lett. 122, 250502 (2019).

[37] F. Vicentini, A. Biella, N. Regnault, and C. Ciuti, Variational Neural-Network Ansatz for Steady States in Open Quantum Systems, Phys. Rev. Lett. 122, 250503 (2019).

[38] A. Nagy and V. Savona, Variational Quantum Monte Carlo Method with a Neural-Network Ansatz for Open Quantum Systems, Phys. Rev. Lett. 122, 250501 (2019).

[39] N. Yoshioka and R. Hamazaki, Constructing neural stationary states for open quantum many-body systems, Phys. Rev. B 99 , 214306 (2019).

[40] U. Schollwöck, The density-matrix renormalization group in the age of matrix product states, Ann. Phys. (NY) 326, 96 (2011).

[41] F. Verstraete, V. Murg, and J. I. Cirac, Matrix product states, projected entangled pair states, and variational renormalization group methods for quantum spin systems, Adv. Phys. 57, 143 (2008). 
[42] W. M. C. Foulkes, L. Mitas, R. J. Needs, and G. Rajagopal, Quantum monte carlo simulations of solids, Rev. Mod. Phys. 73, 33 (2001).

[43] D. Hangleiter, I. Roth, D. Nagaj, and J. Eisert, Easing the Monte Carlo sign problem, Sci. Adv. 6, eabb8341 (2020).

[44] D.-L. Deng, X. Li, and S. Das Sarma, Quantum Entanglement in Neural Network States, Phys. Rev. X 7, 021021 (2017).

[45] I. Goodfellow, Y. Bengio, and A. Courville, Deep Learning (MIT Press, Cambridge, 2016).

[46] X. Yuan, S. Endo, Q. Zhao, Y. Li, and S. C. Benjamin, Theory of variational quantum simulation, Quantum 3, 191 (2019).

[47] S. Endo, J. Sun, Y. Li, S. C. Benjamin, and X. Yuan, Variational Quantum Simulation of General Processes, Phys. Rev. Lett. 125, 010501 (2020).

[48] Z. Hu, R. Xia, and S. Kais, A quantum algorithm for evolving open quantum dynamics on quantum computing devices, Sci. Rep. 10, 1 (2020).

[49] N. Yoshioka, Y. O. Nakagawa, K. Mitarai, and K. Fujii, Variational quantum algorithm for nonequilibrium steady states, Phys. Rev. Research 2, 043289 (2020).

[50] J. R. McClean, S. Boixo, V. N. Smelyanskiy, R. Babbush, and H. Neven, Barren plateaus in quantum neural network training landscapes, Nat. Commun. 9, 4812 (2018).

[51] M. Cerezo, A. Sone, T. Volkoff, L. Cincio, and P. J. Coles, Cost function dependent barren plateaus in shallow parametrized quantum circuits, Nat. Commun. 12, 1791 (2021).

[52] K. Sharma, M. Cerezo, L. Cincio, and P. J. Coles, Trainability of dissipative perceptron-based quantum neural networks, arXiv:2005.12458.

[53] M. Cerezo and P. J. Coles, Impact of barren plateaus on the hessian and higher order derivatives, Quantum Sci. Technol. 6, 035006 (2021).

[54] Z. Holmes, A. Arrasmith, B. Yan, P. J. Coles, A. Albrecht, and A. T. Sornborger, Barren Plateaus Preclude Learning Scramblers, Phys. Rev. Lett. 126, 190501 (2021).

[55] A. Arrasmith, M. Cerezo, P. Czarnik, L. Cincio, and P. J. Coles, Effect of barren plateaus on gradient-free optimization, Quantum 5, 558 (2021).

[56] S. Wang, E. Fontana, M. Cerezo, K. Sharma, A. Sone, L. Cincio, and P. J. Coles, Noise-induced barren plateaus in variational quantum algorithms, Nat. Commun. 12, 6961 (2021).
[57] C. O. Marrero, M. Kieferová, and N. Wiebe, Entanglement induced barren plateaus, PRX Quantum 2, 040316 (2021).

[58] A. Pesah, M. Cerezo, S. Wang, T. Volkoff, A. T. Sornborger, and P. J. Coles, Absence of Barren Plateaus in Quantum Convolutional Neural Networks, Phys. Rev. X 11, 041011 (2021).

[59] S. Sorella, M. Casula, and D. Rocca, Weak binding between two aromatic rings: Feeling the van der Waals attraction by quantum Monte Carlo methods, J. Chem. Phys. 127, 014105 (2007).

[60] J.-G. Liu and L. Wang, Differentiable learning of quantum circuit born machines, Phys. Rev. A 98, 062324 (2018).

[61] A. W. Harrow and J. C. Napp, Low-Depth Gradient Measurements Can Improve Convergence in Variational Hybrid Quantum-Classical Algorithms, Phys. Rev. Lett. 126, 140502 (2021).

[62] E. Bernstein and U. Vazirani, Quantum complexity theory, SIAM J. Comput. 26, 1411 (1997).

[63] S. Aaronson, Quantum computing, postselection, and probabilistic polynomial-time, Proc. R. Soc. A. 461, 3473 (2005).

[64] Y. Han, L. A. Hemaspaandra, and T. Thierauf, Threshold computation and cryptographic security, SIAM J. Comput. 26, 59 (1997).

[65] S. Toda, PP is as hard as the polynomial-time hierarchy, SIAM J. Comput. 20, 865 (1991).

[66] M. J. Bremner, R. Jozsa, and D. J. Shepherd, Classical simulation of commuting quantum computations implies collapse of the polynomial hierarchy, Proc. R. Soc. A. 467, 459 (2011).

[67] X. Gao, S.-T. Wang, and L.-M. Duan, Quantum Supremacy for Simulating a Translation-Invariant Ising Spin Model, Phys. Rev. Lett. 118, 040502 (2017).

[68] M.-D. Choi, Completely positive linear maps on complex matrices, Linear Algebra Appl. 10, 285 (1975).

[69] N. Metropolis, A. W. Rosenbluth, M. N. Rosenbluth, A. H. Teller, and E. Teller, Equation of state calculations by fast computing machines, J. Chem. Phys. 21, 1087 (1953).

[70] A. K. Ekert, C. M. Alves, D. K. L. Oi, M. Horodecki, P. Horodecki, and L. C. Kwek, Direct Estimations of Linear and Nonlinear Functionals of a Quantum State, Phys. Rev. Lett. 88, 217901 (2002)

[71] J. R. Johansson, P. D. Nation, and F. Nori, Qutip: An opensource Python framework for the dynamics of open quantum systems, Comput. Phys. Commun. 183, 1760 (2012). 